\title{
Initial- and Final-State Temperatures of Emission Source from Differential Cross-Section in Squared Momentum Transfer in High-Energy Collisions
}

\author{
Qi Wang, ${ }^{1}$ Fu-Hu Liu $\mathbb{D}^{1},{ }^{1}$ and Khusniddin K. Olimov $\mathbb{D}^{2}$ \\ ${ }^{1}$ Institute of Theoretical Physics \& Collaborative Innovation Center of Extreme Optics \& State Key Laboratory of Quantum Optics \\ and Quantum Optics Devices, Shanxi University, Taiyuan 030006, China \\ ${ }^{2}$ Laboratory of High Energy Physics, Physical-Technical Institute of SPA "Physics-Sun" of Uzbek Academy of Sciences, \\ Chingiz Aytmatov Str. 2b, Tashkent 100084, Uzbekistan
}

Correspondence should be addressed to Fu-Hu Liu; fuhuliu@163.com and Khusniddin K. Olimov; khkolimov@gmail.com

Received 10 February 2021; Revised 30 April 2021; Accepted 21 May 2021; Published 17 June 2021

Academic Editor: Lorenzo Bianchini

Copyright @ 2021 Qi Wang et al. This is an open access article distributed under the Creative Commons Attribution License, which permits unrestricted use, distribution, and reproduction in any medium, provided the original work is properly cited. The publication of this article was funded by SCOAP ${ }^{3}$.

The differential cross-section in squared momentum transfer of $\rho, \rho^{0}, \omega, \phi, f_{0}(980), f_{1}(1285), f_{0}(1370), f_{1}(1420), f_{0}(1500)$, and $J / \psi$ produced in high-energy virtual photon-proton $\left(\gamma^{*} p\right)$, photon-proton $(\gamma p)$, and proton-proton $(p p)$ collisions measured by the H1, ZEUS, and WA102 Collaborations is analyzed by the Monte Carlo calculations. In the calculations, the Erlang distribution, Tsallis distribution, and Hagedorn function are separately used to describe the transverse momentum spectra of the emitted particles. Our results show that the initial- and final-state temperatures increase from lower squared photon virtuality to a higher one and decrease with the increase of center-of-mass energy.

\section{Introduction}

In high-energy collisions, it is interesting for us to describe the excitation and equilibrium degrees of an interacting system because of the two degrees related to the reaction mechanism and evolution process of the collision system [1-10]. In the progress of describing the excitation degree and structure character of the system, temperature is an important quantity in physics in view of intuitiveness and representation. In high-energy collisions, different types of temperature are used [11-18], which usually refer to the initial temperature $T_{i}$, quark-hadron transition temperature $T_{t r}$, chemical freeze-out temperature $T_{c h}$, kinetic freeze-out or final-state temperature ("confinement" temperature) $T_{k i n}$ or $T_{0}$, and effective temperature $T_{\text {eff }}$ or $T$, etc. In this work, we emphatically discuss the initial- and final-state temperatures, though other types of temperatures are also important.

The initial temperature $T_{i}$ is the temperature of emission source or interacting system when a projectile particle or nucleus and a target particle or nucleus undergo the initial stage of a collision. It represents the excitation degree of the emission source or that of an interacting system in the initial state of collisions, and it is usually meant as describing the interacting system after thermalization. The initial temperature $T_{i}$ can be extracted by fitting the transverse momentum $p_{T}$ spectra of particles by using some distributions such as the Erlang distribution [19-21], Tsallis distribution [22, 23], Hagedorn function [24], and Lévy-Tsallis function [25]. Here, both the names of distribution and the function are used according to the various accepted terminologies in the literature, though they represent the similar probability density function in fitting the particle spectra. Meanwhile, the average transverse momentum $\left\langle p_{T}\right\rangle$ can be obtained from the same function.

The final-state temperature $T_{0}$ is usually known as the kinetic freeze-out temperature, which refers to the temperature of emission source when the inelastic collisions ceased and there are only elastic collisions among particles. In the last stage of collisions, the momentum distribution of particles is fixed and the transverse momentum spectra can be 
measured in experiments. The excitation degree of the system in the last stage can be described by the final-state temperature $T_{0}$ in which the influence of flow effect is excluded. The temperature or related main parameters used in the Erlang distribution [19-21], Tsallis distribution [22, 23], Hagedorn function [24], and Lévy-Tsallis function [25] are not $T_{0}$, but the effective temperature $T$ in which the influence of flow effect is not excluded.

The Mandelstam variables [26] consist of the fourmomentum of particles in a two-body reaction. Both the squared momentum transfer and the transverse momentum can represent the kinetic character of particles. Let us use the squared momentum transfer to replace the transverse momentum in fitting the particle spectra. Then, we can fit the squared momentum transfer spectra with the related distributions to obtain the initial temperature $T_{i}$, average transverse momentum $\left\langle p_{T}\right\rangle$, and other quantities. Of course, in fitting the squared momentum transfer spectra, the abovementioned distributions cannot be used directly. In fact, we have to use the Monte Carlo method to obtain the concrete value of a transverse momentum for a given particle from the mentioned distributions. Then, the concrete value of squared momentum transfer can be obtained from the definition.

Except for the temperature parameter, other parameters also describe partly the characters of the interacting system. For instance, the entropy index $q$ which describes the degree of equilibrium can be extracted from the Tsallis distribution $[22,23]$ considering the particle mass. Meanwhile, $q$ can be extracted from the Hagedorn function [24] which is the same as the Lévy-Tsallis function [25] for a particle neglecting its mass. If there is relation between the Tsallis distribution and the Hagedorn function, we may say that the former one covers the latter one in which the mass is neglected. Because the universality, similarity, or common characteristics exist in high-energy collisions [27-36], some distributions used in the large collision system can be also used in a small collision system.

In this paper, the differential cross-section in the squared momentum transfer of $\rho, \rho^{0}, \phi$, and $J / \psi$ produced in virtual photon-proton $\left(\gamma^{*} p\right)$ collisions and $\omega$ and $J / \psi$ produced in photon-proton $(\gamma p)$ collisions, as well as $f_{0}(980), f_{1}(1285)$, $f_{0}(1370), f_{1}(1420)$, and $f_{0}(1500)$ produced in protonproton $(p p)$ collisions measured by the $\mathrm{H} 1[37,38]$, ZEUS [39-42], and WA102 Collaborations $[43,44]$ is fitted with the results from the Monte Carlo calculations. Firstly, the transverse momenta satisfied with the Erlang distribution, Tsallis distribution, and Hagedorn function are generated. Secondly, these special transverse momenta are transformed to the squared momentum transfers. Thirdly and lastly, the distribution of squared momentum transfers is obtained and fitted to the experimental data by the least squares method.

\section{Formalism and Method}

2.1. The Erlang Distribution. The Erlang distribution is the convolution of multiple exponential distributions. In the framework of a multisource thermal model [19-21], we may think that more than one parton (or parton-like) contribute to the transverse momentum of the considered particle. The $j$-th parton (or parton-like) is assumed to contribute to the transverse momentum to be $p_{t j}$ which obeys an exponential distribution with the average $\left\langle p_{t}\right\rangle$ which is $j$-ordinal number independent. We have the probability density function obeyed by $p_{t j}$ to be

$$
f\left(p_{t j}\right)=\frac{1}{\left\langle p_{t}\right\rangle} \exp \left(-\frac{p_{t j}}{\left\langle p_{t}\right\rangle}\right)
$$

The average $\left\langle p_{t}\right\rangle$ reflects the excitation degree of contributor parton and can be regarded as the effective temperature $T$.

The contribution of all $n_{s}$ partons to $p_{T}$ is the sum of various $p_{t j}$. The distribution of $p_{T}$ is then the convolution of $n_{s}$ exponential functions [19-21]. We have the $p_{T}$ distribution (the probability density function of $p_{T}$ ) of final-state particles to be the Erlang distribution

$$
f_{1}\left(p_{T}\right)=\frac{1}{N} \frac{d N}{d p_{T}}=\frac{p_{T}^{n_{s}-1}}{\left(n_{s}-1\right) !\left\langle p_{t}\right\rangle^{n_{s}}} \exp \left(-\frac{p_{T}}{\left\langle p_{t}\right\rangle}\right)
$$

where $N$ denotes the number of all considered particles and $p_{T}$ has an average of $\left\langle p_{T}\right\rangle=\int_{0}^{\infty} p_{T} f_{1}\left(p_{T}\right) d p_{T}=n_{s}\left\langle p_{t}\right\rangle$. Equation (2) is naturally normalized to be 1. In Equation (2), there are two free parameters, $n_{s}$ and $\left\langle p_{t}\right\rangle$.

2.2. The Tsallis Distribution. The Tsallis distribution [22, 23] has more than one form, which are widely used in the field of high-energy collisions. Conveniently, we use the following form

$$
f_{2}\left(p_{T}\right)=\frac{1}{N} \frac{d N}{d p_{T}}=C p_{T}\left(1+\frac{m_{T}-m_{0}}{n T}\right)^{-n}
$$

where $C$ is the normalization constant, $m_{T}=\sqrt{p_{T}^{2}+m_{0}^{2}}$ is the transverse mass, $m_{0}$ is the rest mass, $n=1 /(q-1)$, and $q$ is the entropy index $[22,23]$. Equation (3) is valid only at midrapidity $(y \approx 0)$ which results in $\cosh y \approx 1$ and the particle energy $E=m_{T} \cosh y \approx m_{T}$.

In Equation (3), a large $n$ corresponds to a $q$ that is close to 1 , and the source or system approaches to equilibrium. The larger the parameter $n$ is, the closer to 1 the entropy index $q$ is, with the source or system being at a higher degree of equilibrium. There is no exact minimum $n$ or maximum $q$ [22-25] which is a limit for approximate equilibrium. Empirically, in the case of $n \geq 4$ or $q \leq 1.25$ which is $25 \%$ more than 1 (even $n \geq 5$ or $q \leq 1.2$ which is $20 \%$ more than 1 ), the source or system can be regarded as being in a state of approximate (local) equilibrium. Usually, in high-energy collisions, the source or system is approximately in equilibrium due to $n$ being large enough.

2.3. The Hagedorn Function. The Hagedorn function [24] is an inverse power law which has the probability density 


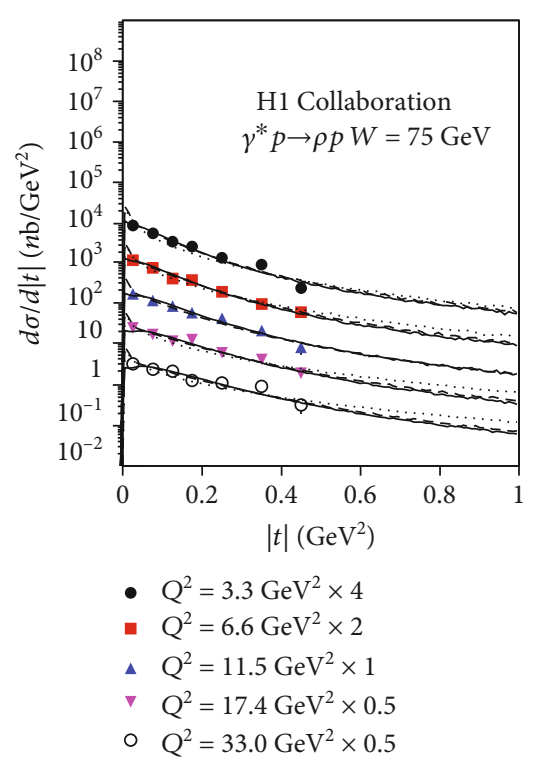

(a)

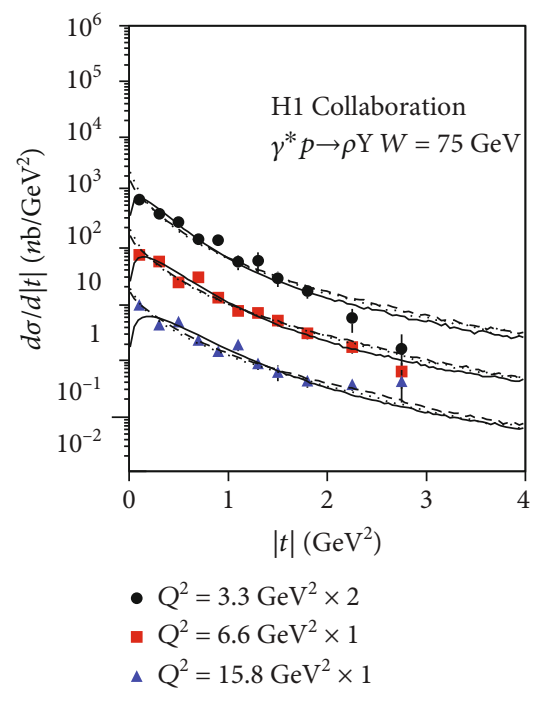

(b)

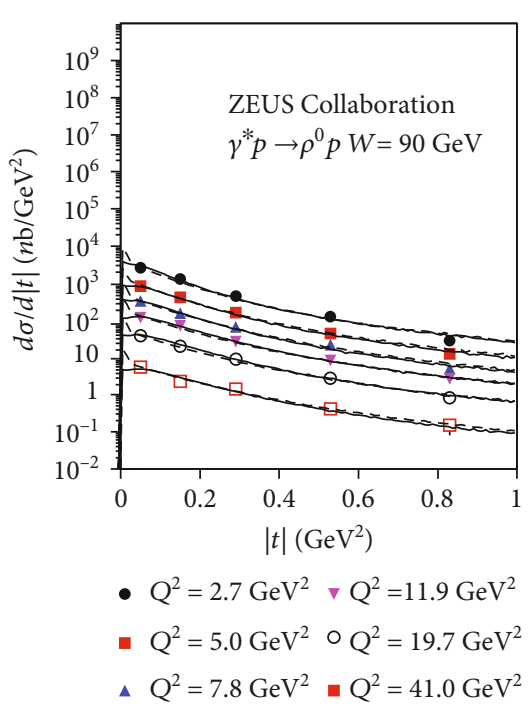

(c)

FIGURE 1: The differential cross-section in squared momentum transfer of (a) $\gamma^{*} p \longrightarrow \rho p$, (b) $\gamma^{*} p \longrightarrow \rho Y$, and (c) $\gamma^{*} p \longrightarrow \rho^{0} p$ produced in $e p$ collisions at $(\mathrm{a}, \mathrm{b}) W=75 \mathrm{GeV}$ and (c) $W=90 \mathrm{GeV}$. The experimental data points from (a, b) nonexclusive and (c) exclusive productions are measured by the H1 [37] and ZEUS Collaborations [39], respectively, with different $Q^{2}$ marked in the panels. The data points are fitted by the Monte Carlo calculations with the Erlang distribution Equation (2) (the solid curves), the Tsallis distribution Equation (3) (the dashed curves), and the Hagedorn function Equation (4) (the dotted curves) for $p_{3 T}$ in Equation (8).

function of $p_{T}$ to be

$$
f_{3}\left(p_{T}\right)=\frac{1}{N} \frac{d N}{d p_{T}}=A p_{T}\left(1+\frac{p_{T}}{p_{0}}\right)^{-n_{0}}
$$

where $A$ is the normalization constant, $n_{0}$ is a free parameter which is similar to $n$ in the Tsallis distribution $[22,23]$, and $p_{0}$ is a free parameter which is similar to the product of $n T$ in the Tsallis distribution. Note here that it appears as if $p_{0}$ $=n T$ is a perfect liquid-like relation; however, $p_{0}$ is a transverse momentum and $n$ is a dimensionless number. This is not meant in a perfect liquid sense, but the letters are just randomly coinciding.

It should be noted that the Hagedorn function is a special case of the Tsallis distribution in which $m_{0}$ can be neglected. Generally, at high $p_{T}$, we may neglect $m_{0}$, observing the two distributions being very similar to each other. At low $p_{T}$, the two distributions have obvious differences due to nonignorable $m_{0}$. To build a connection with the entropy index $q$, we have $n_{0} \approx 1 /(q-1)$. To build a connection with the effective temperature $T$, we have $p_{0} \approx n_{0} T \approx T /(q-1)$.

2.4. The Squared Momentum Transfer. In the center-of-mass reference frame, in a two-body reaction $1+2 \longrightarrow 3+4$ or in a two-body-like reaction, it is supposed that particle 1 is incident along the $z$ direction and particle 2 is incident along the opposite direction. In addition, particle 3 is emitted with angle $\theta$ relative to the $z$ direction and particle 4 is emitted along the opposite direction. According to Ref. [26], three
Mandelstam variables are defined as

$$
\begin{gathered}
s=-\left(P_{1}+P_{2}\right)^{2}=-\left(P_{3}+P_{4}\right)^{2}, \\
t=-\left(P_{1}-P_{3}\right)^{2}=-\left(-P_{2}+P_{4}\right)^{2}, \\
u=-\left(P_{1}-P_{4}\right)^{2}=-\left(-P_{2}+P_{3}\right)^{2},
\end{gathered}
$$

where $P_{1}, P_{2}, P_{3}$, and $P_{4}$ are four-momenta of particles 1, 2, 3, and 4 , respectively.

In the Mandelstam variables, slightly varying the form, $\sqrt{s}$ is the center-of-mass energy, $-t$ is the squared momentum transfer between particles 1 and 3 , and $-u$ is the squared momentum transfer between particles 1 and 4 . Conveniently, let $|t|$ be the squared momentum transfer between particles 1 and 3 . We have

$$
\begin{aligned}
|t| & =\left|\left(E_{1}-E_{3}\right)^{2}-\left(\vec{p}_{1}-\vec{p}_{3}\right)^{2}\right| \\
& =\left|m_{1}^{2}+m_{3}^{2}-2 E_{1} \sqrt{\left(\frac{p_{3 T}}{\sin \theta}\right)^{2}+m_{3}^{2}}+2 \sqrt{E_{1}^{2}-m_{1}^{2}} \frac{p_{3 T}}{\tan \theta}\right| .
\end{aligned}
$$

Here, $E_{1}$ and $E_{3}, \vec{p}_{1}$ and $\vec{p}_{3}$, and $m_{1}$ and $m_{3}$ are the energy, momentum, and rest mass of particles 1 and 3 , respectively. In particular, $p_{3 T}$ is the transverse momentum of particle 3 , which is referred to be perpendicular to the $z$ direction.

As the energy of incoming photon in the center-of-mass reference frame of the reaction, $E_{1}$ in Equation (8) should be a fixed value. However, $E_{1}$ has a slight shift from the peak 
TABLE 1: Values of $E_{1},\left\langle p_{t}\right\rangle, n_{s}, T_{i}$, and $\chi^{2} /$ ndof corresponding to the solid curves in Figures $1-3$, where $n_{s}=5$ that is not listed in the table to avoid trivialness. In some cases, ndof is less than 1, which is denoted by "-" in the last column, and the corresponding curve is only to guide the eyes. For Figure 3(a), the first and second $Q^{2}=6.8 \mathrm{GeV}^{2}$ are averaged from the ranges of $Q^{2}=2-100$ and $5-10 \mathrm{GeV}^{2}$, respectively.

\begin{tabular}{|c|c|c|c|c|c|c|}
\hline Figure & Reaction & Main selection & $E_{1}(\mathrm{GeV})$ & $\left\langle p_{t}\right\rangle(\mathrm{GeV} / \mathrm{c})$ & $T_{i}(\mathrm{GeV})$ & $\chi^{2} /$ ndof \\
\hline \multirow{5}{*}{ Figure 1(a) } & \multirow{5}{*}{$\gamma^{*} p \longrightarrow \rho p$} & $Q^{2}=3.3 \mathrm{GeV}^{2}$ & $0.960 \pm 0.003$ & $0.045 \pm 0.003$ & $0.174 \pm 0.011$ & $17 / 3$ \\
\hline & & 6.6 & $0.960 \pm 0.003$ & $0.048 \pm 0.001$ & $0.186 \pm 0.004$ & $8 / 3$ \\
\hline & & 11.5 & $0.960 \pm 0.001$ & $0.052 \pm 0.001$ & $0.201 \pm 0.003$ & $3 / 3$ \\
\hline & & 17.4 & $0.960 \pm 0.003$ & $0.057 \pm 0.002$ & $0.221 \pm 0.008$ & $9 / 3$ \\
\hline & & 33.0 & $0.960 \pm 0.001$ & $0.061 \pm 0.002$ & $0.236 \pm 0.007$ & $5 / 3$ \\
\hline \multirow{3}{*}{ Figure 1(b) } & \multirow{3}{*}{$\gamma^{*} p \longrightarrow \rho Y$} & $Q^{2}=3.3 \mathrm{GeV}^{2}$ & $0.960 \pm 0.020$ & $0.085 \pm 0.010$ & $0.329 \pm 0.039$ & $47 / 7$ \\
\hline & & 6.6 & $0.960 \pm 0.020$ & $0.100 \pm 0.012$ & $0.387 \pm 0.046$ & $95 / 7$ \\
\hline & & 15.8 & $0.960 \pm 0.020$ & $0.115 \pm 0.020$ & $0.445 \pm 0.078$ & $86 / 7$ \\
\hline \multirow{6}{*}{ Figure 1(c) } & \multirow{6}{*}{$\gamma^{*} p \longrightarrow \rho^{0} p$} & $Q^{2}=2.7 \mathrm{GeV}^{2}$ & $0.961 \pm 0.004$ & $0.047 \pm 0.001$ & $0.182 \pm 0.004$ & $22 / 1$ \\
\hline & & 5.0 & $0.961 \pm 0.001$ & $0.052 \pm 0.001$ & $0.201 \pm 0.003$ & $5 / 1$ \\
\hline & & 7.8 & $0.961 \pm 0.001$ & $0.052 \pm 0.002$ & $0.201 \pm 0.007$ & $11 / 1$ \\
\hline & & 11.9 & $0.961 \pm 0.001$ & $0.055 \pm 0.001$ & $0.213 \pm 0.004$ & $2 / 1$ \\
\hline & & 19.7 & $0.961 \pm 0.001$ & $0.056 \pm 0.002$ & $0.217 \pm 0.008$ & $1 / 1$ \\
\hline & & 41.0 & $0.961 \pm 0.002$ & $0.058 \pm 0.002$ & $0.225 \pm 0.008$ & $3 / 1$ \\
\hline Figure 2(a) & $\gamma p \longrightarrow \omega p$ & $70 \mathrm{GeV}<W<90 \mathrm{GeV}$ & $0.960 \pm 0.010$ & $0.036 \pm 0.005$ & $0.139 \pm 0.019$ & $5 / 2$ \\
\hline \multirow{4}{*}{ Figure 2(b) } & \multirow{3}{*}{$\gamma^{*} p \longrightarrow \phi p$} & $Q^{2}=3.3 \mathrm{GeV}^{2}$ & $0.970 \pm 0.010$ & $0.045 \pm 0.005$ & $0.174 \pm 0.020$ & $22 / 3$ \\
\hline & & 6.6 & $0.970 \pm 0.010$ & $0.048 \pm 0.003$ & $0.186 \pm 0.012$ & $11 / 3$ \\
\hline & & 15.8 & $0.970 \pm 0.010$ & $0.050 \pm 0.002$ & $0.194 \pm 0.008$ & $9 / 3$ \\
\hline & $\gamma^{*} p \longrightarrow \phi Y$ & $W=75 \mathrm{GeV}$ & $0.970 \pm 0.030$ & $0.095 \pm 0.007$ & $0.368 \pm 0.027$ & $4 /-$ \\
\hline \multirow{7}{*}{ Figure 2(c) } & \multirow{7}{*}{$\gamma^{*} p \longrightarrow \phi p$} & $Q^{2}=2.4 \mathrm{GeV}^{2}$ & $0.960 \pm 0.010$ & $0.058 \pm 0.002$ & $0.225 \pm 0.008$ & $4 /-$ \\
\hline & & 3.6 & $0.960 \pm 0.010$ & $0.060 \pm 0.005$ & $0.232 \pm 0.020$ & $7 /-$ \\
\hline & & 5.2 & $0.960 \pm 0.010$ & $0.063 \pm 0.002$ & $0.244 \pm 0.008$ & $4 /-$ \\
\hline & & 6.9 & $0.960 \pm 0.011$ & $0.065 \pm 0.003$ & $0.252 \pm 0.012$ & $6 /-$ \\
\hline & & 9.2 & $0.960 \pm 0.010$ & $0.067 \pm 0.002$ & $0.259 \pm 0.008$ & $7 /-$ \\
\hline & & 12.6 & $0.960 \pm 0.012$ & $0.070 \pm 0.002$ & $0.271 \pm 0.008$ & $5 /-$ \\
\hline & & 19.7 & $0.960 \pm 0.020$ & $0.072 \pm 0.005$ & $0.279 \pm 0.019$ & $5 /-$ \\
\hline \multirow{5}{*}{ Figure 2(d) } & $p p \longrightarrow p p f_{0}(980)$ & & $0.960 \pm 0.001$ & $0.062 \pm 0.002$ & $0.240 \pm 0.008$ & $15 / 2$ \\
\hline & $p p \longrightarrow p p f_{1}(1285)$ & & $1.018 \pm 0.001$ & $0.045 \pm 0.002$ & $0.174 \pm 0.007$ & $4 / 2$ \\
\hline & $p p \longrightarrow p p f_{0}(1370)$ & $\sqrt{s_{N N}}=29.1 \mathrm{GeV}$ & $1.033 \pm 0.002$ & $0.035 \pm 0.001$ & $0.136 \pm 0.004$ & $86 / 2$ \\
\hline & $p p \longrightarrow p p f_{1}(1420)$ & & $1.050 \pm 0.001$ & $0.058 \pm 0.002$ & $0.225 \pm 0.008$ & $21 / 2$ \\
\hline & $p p \longrightarrow p p f_{0}(1500)$ & & $1.056 \pm 0.001$ & $0.063 \pm 0.002$ & $0.244 \pm 0.008$ & $16 / 2$ \\
\hline \multirow{4}{*}{ Figure 3(a) } & \multirow{4}{*}{$\gamma^{*} p \longrightarrow J / \psi p$} & $Q^{2}=3.1 \mathrm{GeV}^{2}$ & $1.690 \pm 0.020$ & $0.020 \pm 0.001$ & $0.077 \pm 0.004$ & $3 /-$ \\
\hline & & 6.8 & $1.690 \pm 0.010$ & $0.022 \pm 0.001$ & $0.085 \pm 0.004$ & $5 /-$ \\
\hline & & 6.8 & $1.690 \pm 0.010$ & $0.022 \pm 0.001$ & $0.085 \pm 0.004$ & $1 /-$ \\
\hline & & 16.0 & $1.690 \pm 0.010$ & $0.023 \pm 0.001$ & $0.089 \pm 0.004$ & $8 /-$ \\
\hline \multirow{4}{*}{ Figure 3(b) } & \multirow{4}{*}{$\gamma p \longrightarrow J / \psi p$} & $Q^{2}=0.05 \mathrm{GeV}^{2}$ & $1.700 \pm 0.005$ & $0.018 \pm 0.001$ & $0.070 \pm 0.004$ & $35 /-$ \\
\hline & & 3.2 & $1.700 \pm 0.001$ & $0.020 \pm 0.001$ & $0.077 \pm 0.004$ & $2 /-$ \\
\hline & & 7.0 & $1.700 \pm 0.004$ & $0.022 \pm 0.002$ & $0.085 \pm 0.008$ & $10 /-$ \\
\hline & & 22.4 & $1.700 \pm 0.001$ & $0.024 \pm 0.002$ & $0.093 \pm 0.008$ & $3 / 4$ \\
\hline
\end{tabular}


TABLe 1: Continued.

\begin{tabular}{|c|c|c|c|c|c|c|}
\hline Figure & Reaction & Main selection & $E_{1}(\mathrm{GeV})$ & $\left\langle p_{t}\right\rangle(\mathrm{GeV} / \mathrm{c})$ & $T_{i}(\mathrm{GeV})$ & $\chi^{2} / \mathrm{ndof}$ \\
\hline \multirow{6}{*}{ Figure 3(c) } & \multirow{6}{*}{$\gamma p \longrightarrow J / \psi p$} & $W=45 \mathrm{GeV}$ & $1.700 \pm 0.012$ & $0.026 \pm 0.004$ & $0.101 \pm 0.016$ & $12 / 1$ \\
\hline & & 55 & $1.700 \pm 0.018$ & $0.024 \pm 0.004$ & $0.093 \pm 0.016$ & $15 / 1$ \\
\hline & & 65 & $1.700 \pm 0.006$ & $0.022 \pm 0.002$ & $0.085 \pm 0.008$ & $14 / 1$ \\
\hline & & 75 & $1.700 \pm 0.015$ & $0.021 \pm 0.002$ & $0.081 \pm 0.008$ & $16 / 1$ \\
\hline & & 85 & $1.700 \pm 0.010$ & $0.020 \pm 0.001$ & $0.077 \pm 0.004$ & $6 / 1$ \\
\hline & & 95 & $1.700 \pm 0.010$ & $0.018 \pm 0.002$ & $0.070 \pm 0.008$ & $5 / 1$ \\
\hline \multirow{5}{*}{ Figure $3(\mathrm{~d})$} & \multirow{5}{*}{$\gamma p \longrightarrow J / \psi p$} & $W=105 \mathrm{GeV}$ & $1.700 \pm 0.006$ & $0.017 \pm 0.001$ & $0.066 \pm 0.004$ & $25 / 1$ \\
\hline & & 119 & $1.700 \pm 0.001$ & $0.016 \pm 0.001$ & $0.062 \pm 0.004$ & $18 / 1$ \\
\hline & & 144 & $1.700 \pm 0.002$ & $0.015 \pm 0.001$ & $0.058 \pm 0.004$ & $12 / 1$ \\
\hline & & 181 & $1.700 \pm 0.002$ & $0.014 \pm 0.001$ & $0.054 \pm 0.004$ & $46 / 1$ \\
\hline & & 251 & $1.700 \pm 0.001$ & $0.013 \pm 0.001$ & $0.050 \pm 0.004$ & $15 / 1$ \\
\hline
\end{tabular}

value due to different experiments and selections. To obtain a good fit, we treat $E_{1}$ as a parameter which is the same or has small difference in the same/similar reactions. $p_{3 T}$ obeys one of Equations (2)-(4) and $\theta$ obeys an isotropic assumption in the center-of-mass reference frame, which will be discussed later in this section. To obtain $|t|$, we may perform the Monte Carlo calculations. Note that we may calculate $|t|$ from two particles, i.e., particles 1 and 3 , but not from one particle. Instead, for one calculation, $|t|$ means the squared momentum transfer in an event. For many calculations, $|t|$ distribution can be obtained from the statistics. For convenience in the description, the transverse momentum and rest mass of particle 3 are also denoted by $p_{T}$ and $m_{0}$, respectively.

Based on the experiments cited from literature [37-44], we have used two main selection factors for the data. (1) The squared photon virtuality $Q^{2}=-P_{\gamma}^{2}$, where $P_{\gamma}$ denotes the four-momentum of the photon. (2) The center-of-mass energy $\sqrt{s}$ or $W$, i.e., $W=\sqrt{s}=\sqrt{-\left(P_{1}+P_{2}\right)^{2}}$. Let $x$ denote the Bjorken scaling variable; one has $W^{2} \simeq Q^{2} / x$.

2.5. The Initial- and Final-State Temperatures. According to Refs. [45-47], in a color string percolation approach, the initial temperature $T_{i}$ can be estimated as

$$
T_{i}=\sqrt{\frac{\left\langle p_{T}^{2}\right\rangle}{2}},
$$

where $\sqrt{\left\langle p_{T}^{2}\right\rangle}$ is the root mean square of $p_{T}$ and $\left\langle p_{T}^{2}\right\rangle=\int_{0}^{\max }$ $p_{T}^{2} f_{1,2,3}\left(p_{T}\right) d p_{T}$. In the expression of the initial temperature, we have used a single string in the cluster for a given particle production [48], though more than two partons or partonslike take possible part in the formation of the string. That is, we have used the color suppression factor $F(\xi)$ to be 1 in the color string percolation model [48]. Other strings, even if they exist, do not affect noticeably the production of a given particle. If other strings are considered, i.e., if we take the minimum $F(\xi)$ to be 0.6 [48], a higher $T_{i}$ can be obtained by multiplying a revised factor, $\sqrt{1 / F(\xi)}=1.291$, in Equation (9).

The extraction of final-state temperature $T_{0}$ is more complex than that of the initial temperature $T_{i}$. Generally, one may introduce the transverse flow velocity $\beta_{T}$ in the considered function and obtain $T_{0}$ and $\beta_{T}$ simultaneously [49-57], in which the effective temperature $T$ no longer appears. Alternatively, the intercept in $T$ versus $m_{0}$ is assumed to be $T_{0}[50,58-63]$, and the slope in $\left\langle p_{T}\right\rangle$ versus $\bar{m}$ is assumed to be $\beta_{T}$ [62-66], where $\bar{m}$ denotes the average energy. However, the alternative method using intercept and slope is not suitable for us due to the fact that the spectra of more than two types of particles (e.g., pions, kaons, and protons) are needed in the extraction which is not our case.

In the $\gamma^{*} p, \gamma p$, and $p p$ collisions discussed in the present work, the flow effect is not considered by us due to the collective effect being small in the two-body process. This means that $T_{0} \approx T$ in the considered processes. Here, $T$ appears as that in Equation (3). Meanwhile, $T$ can be also approximated by $\left\langle p_{t}\right\rangle$ in Equation (1) and $p_{0} / n_{0}$ in Equation (4). Generally, we may regard different distributions or functions as different "thermometers." Just like the Celsius thermometer and the Fahrenheit thermometer, different thermometers measure different temperatures, though they can be transformed from one to another according to conversion rules. Although we may approximately regard $T$ in Equation (3) as $T_{0}$, a smaller $T_{0}$ can be obtained if the flow effect is considered.

As mentioned above, $T=\left\langle p_{t}\right\rangle=\left\langle p_{T}\right\rangle / n_{s}$ in Equation (1) and the Erlang distribution, and $T_{i}=\sqrt{\left\langle p_{T}^{2}\right\rangle / 2}$. We have $T^{2}$ $n_{s}^{2}=2 T_{i}^{2}-\sigma_{p_{T}}^{2}$, so this would mean that $T$ is basically encoded in $\sigma_{p_{T}}^{2}$, the squared variance of $p_{T}$ in the distribution. This also means that $T_{i}$ and $T$ are related through $p_{T}$. It is understandable, because they reflect the violent degrees of collisions at different stages. Generally, $T_{i}>T$; this is natural.

Note that although we may use the final-state temperature, it is not a freeze-out temperature for the small system discussed in this paper. In particular, for $\gamma^{*} p$ and $\gamma p$ reactions, these are just a process describable in terms of 
TABLe 2: Values of $E_{1}, T, n$, and $\chi^{2}$ /ndof corresponding to the dashed curves in Figures 1-3, where “-” in the last column denotes the case of ndof $<1$ and the corresponding curve is only to guide the eyes.

\begin{tabular}{|c|c|c|c|c|c|c|}
\hline Figure & Reaction & Main selection & $E_{1}(\mathrm{GeV})$ & $T(\mathrm{GeV})$ & $n$ & $\chi^{2} /$ ndof \\
\hline \multirow{5}{*}{ Figure 1(a) } & \multirow{5}{*}{$\gamma^{*} p \longrightarrow \rho p$} & $Q^{2}=3.3 \mathrm{GeV}^{2}$ & $0.950 \pm 0.003$ & $0.032 \pm 0.002$ & $18.0 \pm 1.5$ & $27 / 3$ \\
\hline & & 6.6 & $0.950 \pm 0.002$ & $0.037 \pm 0.002$ & $17.5 \pm 2.0$ & $5 / 3$ \\
\hline & & 11.5 & $0.950 \pm 0.002$ & $0.039 \pm 0.001$ & $17.0 \pm 1.0$ & $6 / 3$ \\
\hline & & 17.4 & $0.950 \pm 0.005$ & $0.049 \pm 0.002$ & $16.0 \pm 2.0$ & $6 / 3$ \\
\hline & & 33.0 & $0.950 \pm 0.005$ & $0.054 \pm 0.002$ & $15.0 \pm 2.0$ & $5 / 3$ \\
\hline \multirow{3}{*}{ Figure 1(b) } & \multirow{3}{*}{$\gamma^{*} p \longrightarrow \rho Y$} & $Q^{2}=3.3 \mathrm{GeV}^{2}$ & $0.950 \pm 0.010$ & $0.065 \pm 0.015$ & $5.0 \pm 1.0$ & $55 / 7$ \\
\hline & & 6.6 & $0.950 \pm 0.010$ & $0.075 \pm 0.011$ & $4.5 \pm 1.1$ & $102 / 7$ \\
\hline & & 15.8 & $0.950 \pm 0.010$ & $0.085 \pm 0.020$ & $4.2 \pm 2.0$ & $68 / 7$ \\
\hline \multirow{6}{*}{ Figure 1(c) } & \multirow{6}{*}{$\gamma^{*} p \longrightarrow \rho^{0} p$} & $Q^{2}=2.7 \mathrm{GeV} 2$ & $0.950 \pm 0.002$ & $0.035 \pm 0.001$ & $17.0 \pm 2.0$ & $35 / 1$ \\
\hline & & 5.0 & $0.950 \pm 0.001$ & $0.039 \pm 0.002$ & $16.0 \pm 1.0$ & $8 / 1$ \\
\hline & & 7.8 & $0.950 \pm 0.003$ & $0.041 \pm 0.002$ & $15.0 \pm 0.5$ & $8 / 1$ \\
\hline & & 11.9 & $0.950 \pm 0.002$ & $0.043 \pm 0.002$ & $14.0 \pm 1.0$ & $9 / 1$ \\
\hline & & 19.7 & $0.950 \pm 0.002$ & $0.045 \pm 0.001$ & $13.0 \pm 1.0$ & $2 / 1$ \\
\hline & & 41.0 & $0.950 \pm 0.001$ & $0.047 \pm 0.001$ & $12.0 \pm 1.0$ & $2 / 1$ \\
\hline Figure 2(a) & $\gamma p \longrightarrow \omega p$ & $70 \mathrm{GeV}<W<90 \mathrm{GeV}$ & $0.960 \pm 0.010$ & $0.021 \pm 0.003$ & $20.0 \pm 2.0$ & $5 / 2$ \\
\hline \multirow{4}{*}{ Figure 2(b) } & \multirow{3}{*}{$\gamma^{*} p \longrightarrow \phi p$} & $Q^{2}=3.3 \mathrm{GeV}^{2}$ & $0.970 \pm 0.010$ & $0.025 \pm 0.002$ & $10.0 \pm 1.5$ & $9 / 3$ \\
\hline & & 6.6 & $0.970 \pm 0.010$ & $0.027 \pm 0.003$ & $9.0 \pm 1.0$ & $11 / 3$ \\
\hline & & 15.8 & $0.970 \pm 0.010$ & $0.028 \pm 0.003$ & $8.0 \pm 1.0$ & $23 / 3$ \\
\hline & $\gamma^{*} p \longrightarrow \phi Y$ & $W=75 \mathrm{GeV}$ & $0.970 \pm 0.010$ & $0.060 \pm 0.008$ & $5.0 \pm 1.0$ & $5 /-$ \\
\hline \multirow{7}{*}{ Figure 2(c) } & \multirow{7}{*}{$\gamma^{*} p \longrightarrow \phi p$} & $Q^{2}=2.4 \mathrm{GeV}^{2}$ & $0.961 \pm 0.001$ & $0.040 \pm 0.001$ & $9.0 \pm 0.1$ & $2 /-$ \\
\hline & & 3.6 & $0.961 \pm 0.001$ & $0.041 \pm 0.001$ & $8.3 \pm 0.1$ & $1 /-$ \\
\hline & & 5.2 & $0.961 \pm 0.001$ & $0.043 \pm 0.001$ & $7.4 \pm 0.3$ & $3 /-$ \\
\hline & & 6.9 & $0.961 \pm 0.001$ & $0.043 \pm 0.001$ & $7.2 \pm 0.2$ & $3 /-$ \\
\hline & & 9.2 & $0.961 \pm 0.001$ & $0.045 \pm 0.001$ & $7.0 \pm 0.2$ & $3 /-$ \\
\hline & & 12.6 & $0.961 \pm 0.001$ & $0.046 \pm 0.002$ & $6.7 \pm 0.3$ & $3 /-$ \\
\hline & & 19.7 & $0.961 \pm 0.001$ & $0.048 \pm 0.003$ & $5.4 \pm 0.3$ & $1 /-$ \\
\hline \multirow{5}{*}{ Figure 2(d) } & $p p \longrightarrow p p f_{0}(980)$ & \multirow{5}{*}{$\sqrt{s_{N N}}=29.1 \mathrm{GeV}$} & $0.965 \pm 0.001$ & $0.047 \pm 0.003$ & $8.0 \pm 1.0$ & $9 / 2$ \\
\hline & $p p \longrightarrow p p f_{1}(1285)$ & & $1.017 \pm 0.001$ & $0.021 \pm 0.001$ & $12.6 \pm 0.2$ & $6 / 2$ \\
\hline & $p p \longrightarrow p p f_{0}(1370)$ & & $1.033 \pm 0.001$ & $0.013 \pm 0.001$ & $12.0 \pm 0.3$ & $102 / 2$ \\
\hline & $p p \longrightarrow p p f_{1}(1420)$ & & $1.050 \pm 0.004$ & $0.030 \pm 0.004$ & $9.5 \pm 0.7$ & $17 / 2$ \\
\hline & $p p \longrightarrow p p f_{0}(1500)$ & & $1.062 \pm 0.001$ & $0.034 \pm 0.003$ & $7.0 \pm 0.5$ & $15 / 2$ \\
\hline \multirow{4}{*}{ Figure 3(a) } & \multirow{4}{*}{$\gamma^{*} p \longrightarrow J / \psi p$} & $Q^{2}=3.1 \mathrm{GeV}^{2}$ & $1.680 \pm 0.010$ & $0.0022 \pm 0.0001$ & $22.0 \pm 1.0$ & $6 /-$ \\
\hline & & 6.8 & $1.680 \pm 0.004$ & $0.0024 \pm 0.0001$ & $20.0 \pm 0.3$ & $8 /-$ \\
\hline & & 6.8 & $1.680 \pm 0.004$ & $0.0024 \pm 0.0001$ & $20.0 \pm 0.3$ & $2 /-$ \\
\hline & & 16.0 & $1.680 \pm 0.003$ & $0.0026 \pm 0.0002$ & $16.0 \pm 1.0$ & $9 /-$ \\
\hline \multirow{4}{*}{ Figure 3(b) } & \multirow{4}{*}{$\gamma p \longrightarrow J / \psi p$} & $Q^{2}=0.05 \mathrm{GeV}^{2}$ & $1.685 \pm 0.001$ & $0.0019 \pm 0.0001$ & $25.0 \pm 3.0$ & $33 /-$ \\
\hline & & 3.2 & $1.685 \pm 0.005$ & $0.0020 \pm 0.0001$ & $22.0 \pm 2.0$ & $8 /-$ \\
\hline & & 7.0 & $1.685 \pm 0.002$ & $0.0022 \pm 0.0002$ & $20.0 \pm 2.0$ & $6 /-$ \\
\hline & & 22.4 & $1.685 \pm 0.003$ & $0.0026 \pm 0.0001$ & $16.0 \pm 2.0$ & $3 / 4$ \\
\hline Figure 3(c) & $\gamma p \longrightarrow J / \psi p$ & $W=45 \mathrm{GeV}$ & $1.690 \pm 0.010$ & $0.0042 \pm 0.0001$ & $18.0 \pm 2.0$ & $27 / 1$ \\
\hline
\end{tabular}


TABLE 2: Continued.

\begin{tabular}{|c|c|c|c|c|c|c|}
\hline Figure & Reaction & Main selection & $E_{1}(\mathrm{GeV})$ & $T(\mathrm{GeV})$ & $n$ & $\chi^{2} / \mathrm{ndof}$ \\
\hline & & 55 & $1.690 \pm 0.010$ & $0.0038 \pm 0.0006$ & $19.0 \pm 4.0$ & $35 / 1$ \\
\hline & & 65 & $1.690 \pm 0.010$ & $0.0033 \pm 0.0005$ & $20.0 \pm 3.0$ & $32 / 1$ \\
\hline & & 75 & $1.690 \pm 0.030$ & $0.0030 \pm 0.0007$ & $21.0 \pm 5.0$ & $28 / 1$ \\
\hline & & 85 & $1.690 \pm 0.003$ & $0.0026 \pm 0.0003$ & $22.0 \pm 3.0$ & $18 / 1$ \\
\hline & & 95 & $1.690 \pm 0.007$ & $0.0020 \pm 0.0003$ & $23.0 \pm 3.0$ & $12 / 1$ \\
\hline \multirow{5}{*}{ Figure 3(d) } & \multirow{5}{*}{$\gamma p \longrightarrow J / \psi p$} & $W=105 \mathrm{GeV}$ & $1.690 \pm 0.003$ & $0.0013 \pm 0.0003$ & $24.0 \pm 3.0$ & $34 / 1$ \\
\hline & & 119 & $1.690 \pm 0.001$ & $0.0012 \pm 0.0004$ & $25.0 \pm 2.0$ & $23 / 1$ \\
\hline & & 144 & $1.690 \pm 0.005$ & $0.0011 \pm 0.0004$ & $26.0 \pm 3.0$ & $20 / 1$ \\
\hline & & 181 & $1.690 \pm 0.001$ & $0.0010 \pm 0.0003$ & $27.0 \pm 3.0$ & $40 / 1$ \\
\hline & & 251 & $1.690 \pm 0.001$ & $0.0009 \pm 0.0003$ & $28.0 \pm 2.0$ & $17 / 1$ \\
\hline
\end{tabular}

perturbative quantum chromodynamics ( $\mathrm{pQCD}$ ) and factorization [67], but not a process in which deconfinement or freeze-out is involved. The meaning of the final-state temperature for the large system such as heavy-ion collisions or the small system such as $p p$ collisions with high multiplicity is somehow different from here. At least, for the large system, we may consider the deconfinement- or freeze-out-involved picture. Meanwhile, the flow effect in the large system cannot be neglected.

2.6. The Process of Monte Carlo Calculations. In an analytical calculation, the function Equations (2)-(4) on $p_{T}$ distribution are difficult to use in Equation (8) to obtain the $|t|$ distribution. Instead, we may perform the Monte Carlo calculations. Let $R_{1,2}$ and $r_{1,2,3, \cdots, n_{s}}$ be random numbers distributed evenly in $[0,1]$. To use Equation (8), we have to know the changeable $p_{3 T}$ (i.e., $p_{T}$ ) and $\theta$. Other quantities such as $E_{1}, m_{1}$, and $m_{3}$ in the equation are fixed, though $E_{1}$ is treated by us as a parameter with slight variety.

To obtain a concrete value of $p_{T}$, we need one of Equations (2)-(4). Solving the equation

$$
\int_{0}^{p_{T}} f_{i}\left(p_{T}^{\prime}\right) d p_{T}^{\prime}<R_{1}<\int_{0}^{p_{T}+\delta p_{T}} f_{i}\left(p_{T}^{\prime}\right) d p_{T}^{\prime}
$$

where $i=1,2$, and 3 and $\delta p_{T}$ is a small shift relative to $p_{T}$; we may obtain concrete $p_{T}$. It seems that Equation (10) directly means that the integral of $f_{1}\left(p_{T}\right), f_{2}\left(p_{T}\right)$, and $f_{3}\left(p_{T}\right)$ is the same for the $\left[0, p_{T}\right]$ interval, which essentially means that the three functions are equal (except for a null measure set). In fact, the three functions are different in forms because of Equations (2)-(4), and we need to distinguish them.

In particular, for $f_{1}\left(p_{T}\right)$, we have a simpler expression. Let us solve the equation

$$
\int_{0}^{p_{t j}} f\left(p_{t j}^{\prime}\right) d p_{t j}^{\prime}=r_{j} \quad\left(j=1,2,3, \cdots, n_{s}\right) .
$$

We have

$$
p_{t j}=-\left\langle p_{t}\right\rangle \ln r_{j} \quad\left(j=1,2,3, \cdots, n_{s}\right),
$$

due to Equation (1) being used, where $r_{j}$ in Equation (12) replaced $1-r_{j}$ because both of them are random numbers in $[0,1]$. The simpler expression is

$$
p_{T}=-\left\langle p_{t}\right\rangle \prod_{j=1}^{n_{s}} \ln r_{j}
$$

due to $p_{T}$ being the sum of $n_{s}$ random $p_{t j}$.

To obtain a concrete value of $\theta$, we need the function

$$
f_{\theta}(\theta)=\frac{1}{2} \sin \theta
$$

which is obeyed by $\theta$ under the assumption of isotropic emission in the center-of-mass reference frame. Solving the equation

$$
\int_{0}^{\theta} f_{\theta}\left(\theta^{\prime}\right) d \theta^{\prime}=R_{2}
$$

we have

$$
\theta=2 \arcsin \left(\sqrt{R_{2}}\right),
$$

which is needed by us.

According to the concrete values of $p_{T}$ and $\theta$, and using other quantities, the value of $|t|$ can be obtained from Equation (8). After repeating the calculations many times, the distribution of $|t|$ is obtained statistically. Based on the method of least squares, the related parameters are obtained naturally. Meanwhile, $T_{i}$ can be obtained from Equation (9). $\left\langle p_{T}\right\rangle$ and $\left\langle p_{T}^{2}\right\rangle$ can be obtained from one of Equations (2)-(4) or from the statistics. The errors of parameters are obtained by the general method of statistical simulation. 
TABLe 3: Values of $E_{1}, p_{0}, n_{0}$, and $\chi^{2} /$ ndof corresponding to the dotted curves in Figures $1-3$, where “-” in the last column denotes the case of ndof $<1$ and the corresponding curve is only to guide the eyes.

\begin{tabular}{|c|c|c|c|c|c|c|}
\hline Figure & Reaction & Main selection & $E_{1}(\mathrm{GeV})$ & $p_{0}(\mathrm{GeV} / \mathrm{c})$ & $n_{0}$ & $\chi^{2} /$ ndof \\
\hline \multirow{5}{*}{ Figure 1(a) } & \multirow{5}{*}{$\gamma^{*} p \longrightarrow \rho p$} & $Q^{2}=3.3 \mathrm{GeV}^{2}$ & $0.960 \pm 0.010$ & $1.70 \pm 0.11$ & $19.0 \pm 1.5$ & $66 / 3$ \\
\hline & & 6.6 & $0.960 \pm 0.003$ & $1.85 \pm 0.02$ & $18.0 \pm 0.8$ & $19 / 3$ \\
\hline & & 11.5 & $0.960 \pm 0.007$ & $1.90 \pm 0.10$ & $17.5 \pm 2.0$ & $16 / 3$ \\
\hline & & 17.4 & $0.960 \pm 0.007$ & $2.02 \pm 0.05$ & $15.8 \pm 0.8$ & $16 / 3$ \\
\hline & & 33.0 & $0.960 \pm 0.005$ & $2.12 \pm 0.10$ & $15.0 \pm 1.0$ & $9 / 3$ \\
\hline \multirow{3}{*}{ Figure 1(b) } & \multirow{3}{*}{$\gamma^{*} p \longrightarrow \rho Y$} & $Q^{2}=3.3 \mathrm{GeV}^{2}$ & $0.960 \pm 0.020$ & $2.10 \pm 0.37$ & $15.0 \pm 2.7$ & $78 / 7$ \\
\hline & & 6.6 & $0.960 \pm 0.020$ & $2.30 \pm 0.17$ & $14.0 \pm 0.9$ & $118 / 7$ \\
\hline & & 15.8 & $0.960 \pm 0.020$ & $2.50 \pm 0.27$ & $13.5 \pm 1.0$ & $74 / 7$ \\
\hline \multirow{6}{*}{ Figure 1(c) } & \multirow{6}{*}{$\gamma^{*} p \longrightarrow \rho^{0} p$} & $Q^{2}=2.7 \mathrm{GeV}^{2}$ & $0.950 \pm 0.003$ & $1.59 \pm 0.10$ & $18.5 \pm 0.5$ & $55 / 1$ \\
\hline & & 5.0 & $0.950 \pm 0.001$ & $1.61 \pm 0.04$ & $18.0 \pm 0.5$ & $34 / 1$ \\
\hline & & 7.8 & $0.950 \pm 0.002$ & $1.63 \pm 0.04$ & $17.0 \pm 1.0$ & $33 / 1$ \\
\hline & & 11.9 & $0.950 \pm 0.003$ & $1.63 \pm 0.10$ & $16.5 \pm 1.0$ & $29 / 1$ \\
\hline & & 19.7 & $0.950 \pm 0.002$ & $1.63 \pm 0.05$ & $16.0 \pm 1.3$ & $16 / 1$ \\
\hline & & 41.0 & $0.950 \pm 0.002$ & $1.65 \pm 0.05$ & $15.8 \pm 0.4$ & $2 / 1$ \\
\hline Figure 2(a) & $\gamma p \longrightarrow \omega p$ & $70 \mathrm{GeV}<W<90 \mathrm{GeV}$ & $0.960 \pm 0.010$ & $1.30 \pm 0.05$ & $21.0 \pm 2.0$ & $7 / 2$ \\
\hline \multirow{4}{*}{ Figure 2(b) } & \multirow{3}{*}{$\gamma^{*} p \longrightarrow \phi p$} & $Q^{2}=3.3 \mathrm{GeV}^{2}$ & $0.970 \pm 0.010$ & $1.75 \pm 0.05$ & $20.0 \pm 2.0$ & $12 / 3$ \\
\hline & & 6.6 & $0.970 \pm 0.010$ & $1.80 \pm 0.04$ & $19.0 \pm 1.0$ & $22 / 3$ \\
\hline & & 15.8 & $0.970 \pm 0.010$ & $1.85 \pm 0.03$ & $18.0 \pm 1.0$ & $37 / 3$ \\
\hline & $\gamma^{*} p \longrightarrow \phi Y$ & $W=75 \mathrm{GeV}$ & $0.970 \pm 0.020$ & $1.50 \pm 0.20$ & $11.0 \pm 1.0$ & $6 /-$ \\
\hline \multirow{7}{*}{ Figure 2(c) } & \multirow{7}{*}{$\gamma^{*} p \longrightarrow \phi p$} & $Q^{2}=2.4 \mathrm{GeV}^{2}$ & $0.962 \pm 0.002$ & $2.20 \pm 0.03$ & $16.2 \pm 0.4$ & $10 /-$ \\
\hline & & 3.6 & $0.962 \pm 0.002$ & $2.22 \pm 0.02$ & $16.0 \pm 0.5$ & $5 /-$ \\
\hline & & 5.2 & $0.962 \pm 0.002$ & $2.24 \pm 0.04$ & $15.7 \pm 0.8$ & $4 /-$ \\
\hline & & 6.9 & $0.962 \pm 0.002$ & $2.26 \pm 0.05$ & $15.5 \pm 0.5$ & $5 /-$ \\
\hline & & 9.2 & $0.962 \pm 0.001$ & $2.29 \pm 0.03$ & $15.0 \pm 0.3$ & $7 /-$ \\
\hline & & 12.6 & $0.962 \pm 0.001$ & $2.31 \pm 0.03$ & $14.7 \pm 0.2$ & $5 /-$ \\
\hline & & 19.7 & $0.962 \pm 0.001$ & $2.34 \pm 0.02$ & $14.4 \pm 0.2$ & $1 /-$ \\
\hline \multirow{5}{*}{ Figure 2(d) } & $p p \longrightarrow p p f_{0}(980)$ & $\sqrt{s_{N N}}=29.1 \mathrm{GeV}$ & $0.971 \pm 0.001$ & $1.53 \pm 0.10$ & $13.5 \pm 0.7$ & $56 / 2$ \\
\hline & $p p \longrightarrow p p f_{1}(1285)$ & & $1.023 \pm 0.001$ & $2.00 \pm 0.10$ & $22.0 \pm 0.5$ & $12 / 2$ \\
\hline & $p p \longrightarrow p p f_{0}(1370)$ & & $1.036 \pm 0.002$ & $1.30 \pm 0.10$ & $21.0 \pm 2.0$ & $132 / 2$ \\
\hline & $p p \longrightarrow p p f_{1}(1420)$ & & $1.049 \pm 0.003$ & $2.10 \pm 0.20$ & $16.0 \pm 1.5$ & $21 / 2$ \\
\hline & $p p \longrightarrow p p f_{0}(1500)$ & & $1.065 \pm 0.001$ & $2.30 \pm 0.20$ & $16.0 \pm 1.0$ & $20 / 2$ \\
\hline \multirow{4}{*}{ Figure 3(a) } & \multirow{4}{*}{$\gamma^{*} p \longrightarrow J / \psi p$} & $Q^{2}=3.1 \mathrm{GeV}^{2}$ & $1.678 \pm 0.001$ & $1.25 \pm 0.05$ & $28.0 \pm 1.0$ & $5 /-$ \\
\hline & & 6.8 & $1.678 \pm 0.001$ & $1.29 \pm 0.02$ & $26.0 \pm 3.0$ & $12 /-$ \\
\hline & & 6.8 & $1.678 \pm 0.001$ & $1.29 \pm 0.02$ & $26.0 \pm 3.0$ & $4 /-$ \\
\hline & & 16.0 & $1.678 \pm 0.001$ & $1.31 \pm 0.02$ & $24.0 \pm 1.0$ & $12 /-$ \\
\hline \multirow{4}{*}{ Figure 3(b) } & \multirow{4}{*}{$\gamma p \longrightarrow J / \psi p$} & $Q^{2}=0.05 \mathrm{GeV}^{2}$ & $1.700 \pm 0.001$ & $1.20 \pm 0.02$ & $29.0 \pm 0.5$ & $64 /-$ \\
\hline & & 3.2 & $1.700 \pm 0.001$ & $1.30 \pm 0.02$ & $27.0 \pm 0.7$ & $10 /-$ \\
\hline & & 7.0 & $1.700 \pm 0.001$ & $1.40 \pm 0.03$ & $25.0 \pm 2.0$ & $5 /-$ \\
\hline & & 22.4 & $1.700 \pm 0.001$ & $1.50 \pm 0.01$ & $23.0 \pm 0.1$ & $1 / 4$ \\
\hline Figure 3(c) & $\gamma p \longrightarrow J / \psi p$ & $W=45 \mathrm{GeV}$ & $1.680 \pm 0.003$ & $1.38 \pm 0.10$ & $22.0 \pm 3.0$ & $19 / 1$ \\
\hline
\end{tabular}


TABLe 3: Continued.

\begin{tabular}{|c|c|c|c|c|c|c|}
\hline Figure & Reaction & Main selection & $E_{1}(\mathrm{GeV})$ & $p_{0}(\mathrm{GeV} / \mathrm{c})$ & $n_{0}$ & $\chi^{2} /$ ndof \\
\hline & & 55 & $1.680 \pm 0.010$ & $1.33 \pm 0.20$ & $23.0 \pm 4.0$ & $26 / 1$ \\
\hline & & 65 & $1.680 \pm 0.010$ & $1.29 \pm 0.17$ & $24.0 \pm 4.0$ & $21 / 1$ \\
\hline & & 75 & $1.680 \pm 0.008$ & $1.27 \pm 0.10$ & $24.0 \pm 3.0$ & $21 / 1$ \\
\hline & & 85 & $1.680 \pm 0.002$ & $1.25 \pm 0.15$ & $25.0 \pm 3.0$ & $21 / 1$ \\
\hline & & 95 & $1.680 \pm 0.002$ & $1.21 \pm 0.10$ & $26.0 \pm 3.0$ & $13 / 1$ \\
\hline \multirow{5}{*}{ Figure 3(d) } & \multirow{5}{*}{$\gamma p \longrightarrow J / \psi p$} & $W=105 \mathrm{GeV}$ & $1.680 \pm 0.001$ & $1.18 \pm 0.06$ & $27.0 \pm 1.0$ & $33 / 1$ \\
\hline & & 119 & $1.680 \pm 0.001$ & $1.15 \pm 0.12$ & $28.0 \pm 1.0$ & $25 / 1$ \\
\hline & & 144 & $1.680 \pm 0.001$ & $1.12 \pm 0.11$ & $29.0 \pm 2.0$ & $22 / 1$ \\
\hline & & 181 & $1.680 \pm 0.001$ & $1.11 \pm 0.07$ & $30.0 \pm 1.0$ & $41 / 1$ \\
\hline & & 251 & $1.680 \pm 0.001$ & $1.07 \pm 0.07$ & $31.0 \pm 3.0$ & $13 / 1$ \\
\hline
\end{tabular}

\section{Results and Discussion}

3.1. Comparison with Data. Figure 1 shows the differential cross-section in squared momentum transfer, $d \sigma / d|t|$, of (a) $\gamma^{*} p \longrightarrow \rho p$, (b) $\gamma^{*} p \longrightarrow \rho Y$, and (c) $\gamma^{*} p \longrightarrow \rho^{0} p$ produced in electron-proton (ep) collisions at photon-proton center-of-mass energy (a, b) $W=75 \mathrm{GeV}$ and (c) $W=90$ $\mathrm{GeV}$, where $\sigma$ denotes the cross-section and $Y$ in Figure 1(b) denotes an "elastic" scattering proton or a diffractively excited "proton dissociation" [37]. The experimental data points from $(\mathrm{a}, \mathrm{b})$ nonexclusive and (c) exclusive productions are measured by the $\mathrm{H} 1$ [37] and ZEUS Collaborations [39], respectively, with different average squared photon virtuality (a) $Q^{2}=3.3,6.6,11.5,17.4$, and 33.0 $\mathrm{GeV}^{2}$; (b) $Q^{2}=3.3,6.6$, and $15.8 \mathrm{GeV}^{2}$; and (c) $Q^{2}=2.7$, $5.0,7.8,11.9,19.7$, and $41.0 \mathrm{GeV}^{2}$. The data points are fitted by the Monte Carlo calculations with the Erlang distribution Equation (2) (the solid curves), the Tsallis distribution Equation (3) (the dashed curves), and the Hagedorn function Equation (4) (the dotted curves) for $p_{3 T}$ in Equation (8). Some data are scaled by different quantities marked in the panels for clear visibility. In the calculations, the method of least squares is used to obtain the parameter values. The values of $E_{1},\left\langle p_{t}\right\rangle, n_{s}, T, T, n, p_{0}$, and $n_{0}$ are listed in Tables 1-3 with $\chi^{2}$ and number of degree of freedom (ndof). One can see that in most cases, the calculations based on Equation (8) with Equations (2)-(4) for $p_{3 T}$ can fit approximately the experimental data measured by the $\mathrm{H} 1$ and ZEUS Collaborations.

Figure 2 presents the differential cross-section in squared momentum transfer, $d \sigma / d|t|$, of (a) $\gamma p \longrightarrow \omega p$, (b) $\gamma^{*} p$ $\longrightarrow \phi p$ and $\gamma^{*} p \longrightarrow \phi Y$, (c) $\gamma^{*} p \longrightarrow \phi p$, and (d) $p p \longrightarrow p p$ $V\left(V=f_{0}(980), f_{1}(1285), f_{0}(1370), f_{1}(1420)\right.$, and $\left.f_{0}(1500)\right)$ produced in (a-c) ep and (d) $p p$ collisions in (a) $70 \mathrm{GeV}<$ $W<90 \mathrm{GeV}$, at (b, c) $W=75 \mathrm{GeV}$, and at (d) protonproton center-of-mass energy per nucleon pair $\sqrt{s_{N N}}=29.1$ $\mathrm{GeV}$. The experimental data points from (a, c) exclusive, (b) nonexclusive, and (d) exclusive productions are measured by the ZEUS [40, 41], H1 [37], and WA102 Collaborations $[43,44]$, respectively, with different $Q^{2}$ for only Figure 2(b)
$\left(Q^{2}=3.3,5,6.6\right.$, and $\left.15.8 \mathrm{GeV}^{2}\right)$ and Figure $2(\mathrm{c})\left(Q^{2}=2.4\right.$, 3.6, 5.2, 6.9, 9.2, 12.6, and $19.7 \mathrm{GeV}^{2}$ ). Similar to Figure 1, the data points are fitted by the Monte Carlo calculations based on Equation (8). The values of parameters are listed in Tables $1-3$ with $\chi^{2} /$ ndof. One can see that in most cases, the calculations based on Equation (8) with Equations (2)-(4) for $p_{3 T}$ can fit approximately the experimental data measured by the $\mathrm{H} 1$ and ZEUS Collaborations.

Figure 3 displays the differential cross-section in squared momentum transfer, $d \sigma / d|t|$, of (a) $\gamma^{*} p \longrightarrow J / \psi p$ and (bd) $\gamma p \longrightarrow J / \psi p$ produced in $e p$ collisions at (a) $W=90 \mathrm{GeV}$, in (b) $40 \mathrm{GeV}<W<160 \mathrm{GeV}$, and at (c, d) $Q^{2}=0.05 \mathrm{GeV}^{2}$. The experimental data points from (a) exclusive and $(b-d)$ nonexclusive productions are measured by the ZEUS [42] and $\mathrm{H} 1$ Collaborations [38], respectively, with (a) $Q^{2}=3.1$, 6.8 averaged in 2-100, 6.8 averaged in $5-10$, and $16 \mathrm{GeV}^{2}$ and (b) $Q^{2}=0.05,3.2,7.0$, and $22.4 \mathrm{GeV}^{2}$, as well as with (c) $W=45,55,65,75,85$, and $95 \mathrm{GeV}$ and (d) $W=105$, $119,144,181$, and $251 \mathrm{GeV}$. Similar to Figures 1 and 2, the data points are fitted by the Monte Carlo calculations based on Equation (8). The values of parameters are listed in Tables 1-3 with $\chi^{2} /$ ndof. One can see that in most cases, the calculations based on Equation (8) with Equations (2)-(4) for $p_{3 T}$ can fit approximately the experimental data measured by the $\mathrm{H} 1$ and ZEUS Collaborations.

From the above comparisons, we see that some fits have large $\chi^{2}$ compared to ndof, corresponding to low confidence levels. The parameters obtained from these fits are not representing the data well. We would like to say here that these values are used only for the qualitative description of the data tendencies, but not the quantitative interpretation of the data size. In some cases, ndof $<1$, which means that there were at least as many parameters as data points. This means that a perfect fit should have been found. However, this was not the case here. The reason is that we have used given functions, but not any function such as a polynomial.

3.2. Tendency of Parameters. The dependencies of energy $E_{1}$ of particle 1 on rest mass $m_{0}$ of particle 3 for different two-body reactions are given in Figure 4, where 


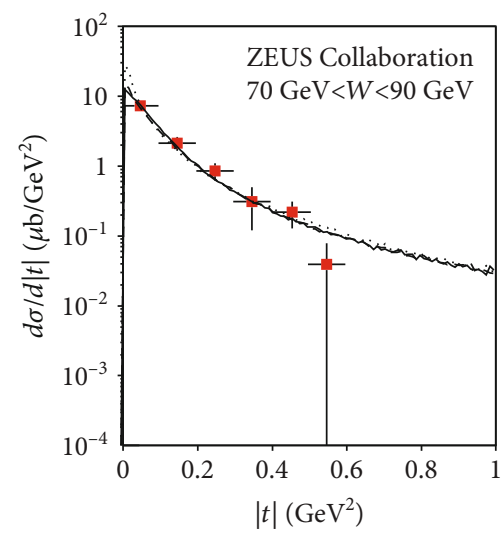

- $\gamma p \rightarrow \omega p$

(a)

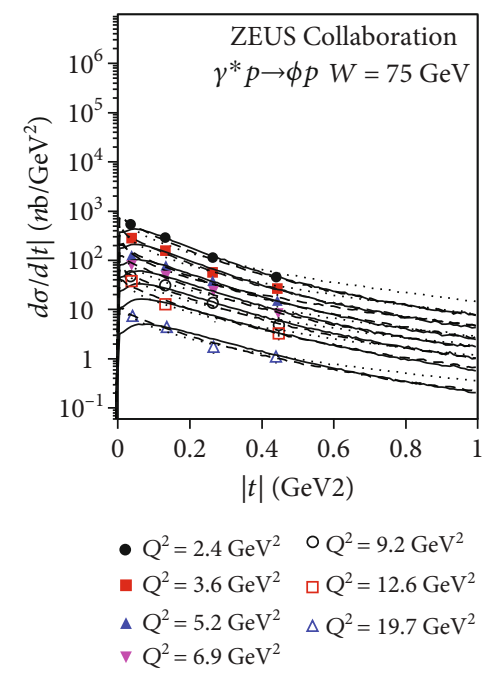

(c)

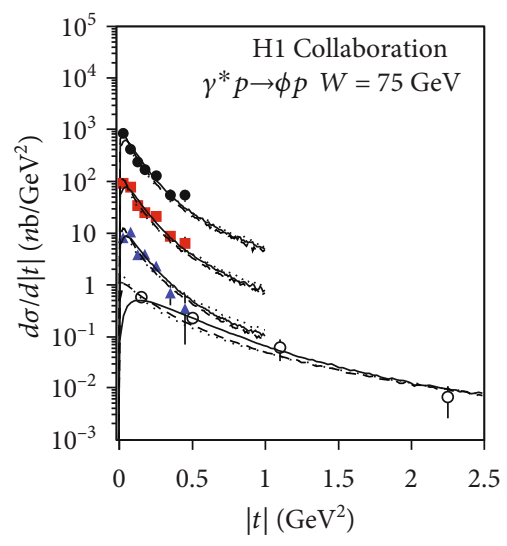

- $\mathrm{Q}^{2}=3.3 \mathrm{GeV}^{2} \times 2$

- $\mathrm{Q}^{2}=6.6 \mathrm{GeV}^{2} \times 1$

\ $\mathrm{Q}^{2}=15.8 \mathrm{GeV}^{2} \times 1$ $\gamma^{*} p \rightarrow \phi \mathrm{Y}$

○ $\mathrm{Q}^{2}=5 \mathrm{GeV}^{2} \times 10^{-2}$

(b)

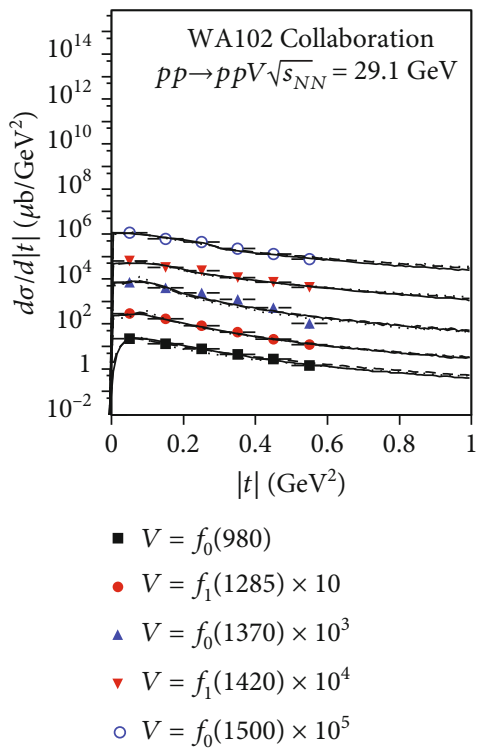

(d)

FiguRE 2: The differential cross-section in squared momentum transfer of (a) $\gamma p \longrightarrow \omega p$, (b) $\gamma^{*} p \longrightarrow \phi p$ and $\gamma^{*} p \longrightarrow \phi Y$, (c) $\gamma^{*} p \longrightarrow \phi p$, and (d) $p p \longrightarrow p p V\left(V=f_{0}(980), f_{1}(1285), f_{0}(1370), f_{1}(1420)\right.$, and $\left.f_{0}(1500)\right)$ produced in (a-c) $e p$ and (d) $p p$ collisions in $($ a) $70 \mathrm{GeV}<W<$ $90 \mathrm{GeV}$, at $(\mathrm{b}, \mathrm{c}) W=75 \mathrm{GeV}$, and at (d) $\sqrt{s_{N N}}=29.1 \mathrm{GeV}$. The experimental data points from (a, c) exclusive, (b) nonexclusive, and (d) exclusive productions are measured by the ZEUS [40, 41], H1 [37], and WA102 Collaborations [43, 44], respectively, with different $Q^{2}$ for only (b) and (c). Similar to Figure 1, the data points are fitted by the Monte Carlo calculations based on Equation (8).

Figures 4(a)-4(c) correspond to the results from the Erlang distribution, Tsallis distribution, and Hagedorn function, respectively. The types of reactions are marked in the panels. Different symbols represent the results from different reactions or collaborations. One can see that the production of particle 3 with larger $m_{0}$ needs the participation of particle 1 with larger $E_{1}$.

The tendency of $E_{1}$ versus $m_{0}$ presented in Figure 4 is natural due to the conservation of energy. The results from the three distributions or functions are almost the same, if not equal to each other, due to the same experimental data considered. In fact, $E_{1}$ should be a fixed value for a given reaction in the present work. However, because different selections such as different $Q^{2}$ and $W$ are used in experiments, $E_{1}$ has a slight shift from the peak value. Thus, we may regard $E_{1}$ as a parameter and obtain it from the fits.

The dependencies of (a) $\left\langle p_{T}\right\rangle$, (b) $T_{i}$, (c) $T_{0}$, (d) $n$, (e) $p_{0}$, and (f) $n_{0}$ on average squared photon virtuality $Q^{2}$ for different two-body reactions are shown in Figure 5. The types of reactions are marked in the panels. Different symbols for 


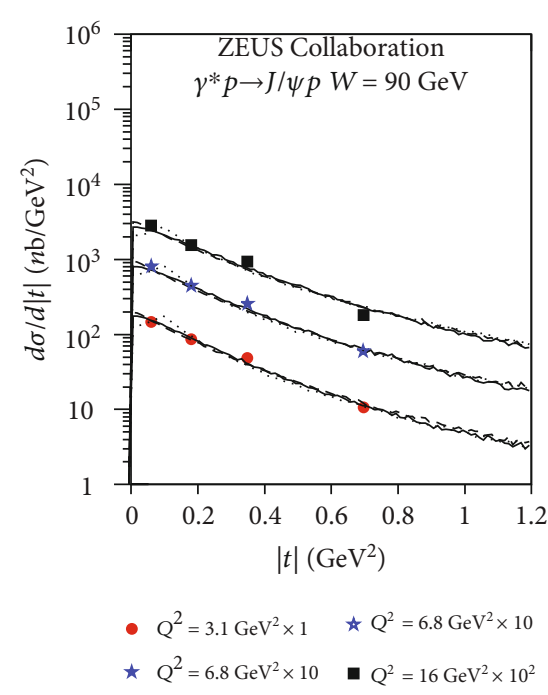

(a)

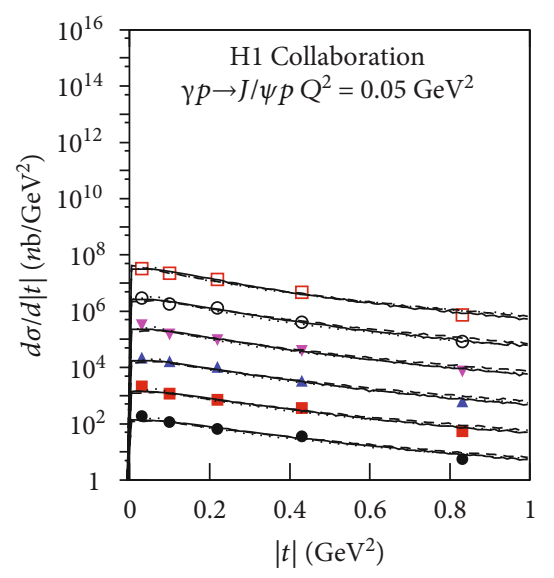

- $W=45 \mathrm{GeV}$

- $W=55 \mathrm{GeV} \times 10$

^ $W=65 \mathrm{GeV} \times 10^{2}$

v $W=75 \mathrm{GeV} \times 10^{3}$

○ $W=85 \mathrm{GeV} \times 10^{4}$

ㅁ $=95 \mathrm{GeV} \times 10^{5}$

(c)

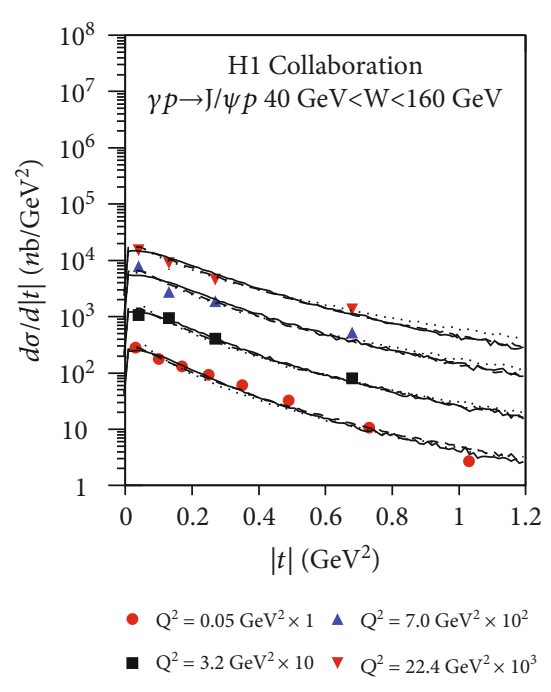

(b)

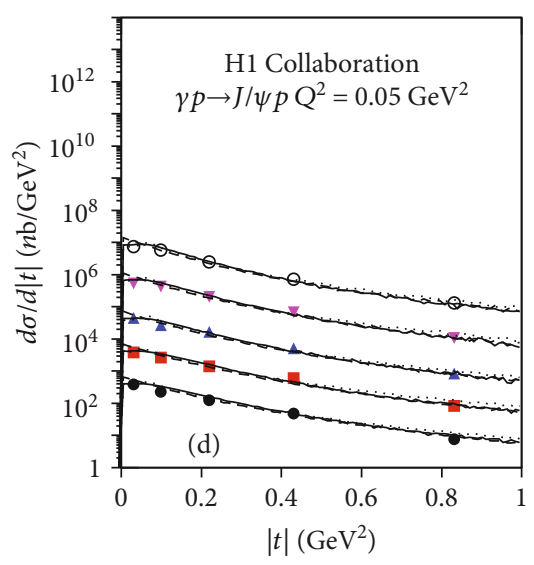

- $W=105 \mathrm{GeV} \times 1$

- $W=119 \mathrm{GeV} \times 10$

^ $W=144 \mathrm{GeV} \times 10^{2}$

จ $W=181 \mathrm{GeV} \times 10^{3}$

○ $W=251 \mathrm{GeV} \times 10^{4}$

(d)

FIGURE 3: The differential cross-section in squared momentum transfer of (a) $\gamma^{*} p \longrightarrow J / \psi p$ and (b-d) $\gamma p \longrightarrow J / \psi p$ produced in $e p$ collisions at (a) $W=90 \mathrm{GeV}$, in (b) $40 \mathrm{GeV}<W<160 \mathrm{GeV}$, and at (c, d) $Q^{2}=0.05 \mathrm{GeV}^{2}$. The experimental data points from (a) exclusive and (b-d) nonexclusive productions are measured by the ZEUS [42] and H1 Collaborations [38], respectively, with different $Q^{2}$ marked in panels (a) and (b), as well as with different $W$ marked in (c) and (d), where in (a), the first and second $Q^{2}=6.8 \mathrm{GeV}^{2}$ are averaged from the ranges of $Q^{2}=2-100$ and 5-10 GeV ${ }^{2}$, respectively. Similar to Figures 1 and 2, the data points are fitted by the Monte Carlo calculations based on Equation (8).

different reactions represent the parameter values extracted from Figures 1-3 and listed in Tables 1-3, where the Erlang distribution, Tsallis distribution, and Hagedorn function in the ranges of available data are used. In particular, $\left\langle p_{T}\right\rangle=n_{s}$ $\left\langle p_{t}\right\rangle$ from Table 1 and $T_{0}=T$ from Table 2 . One can see that $\left\langle p_{T}\right\rangle, T_{i}, T_{0}$, and $p_{0}$ increase generally with increases in $Q^{2}$, and $n$ and $n_{0}$ decrease significantly with an increase in $Q^{2}$.

Because of $Q^{2}$ being a reflection of a hard scale of reaction, this is natural that a harder scale results in a higher excitation degree and then a larger $\left\langle p_{T}\right\rangle, T_{i}$, and $T_{0}$. In most cases, one can see a large enough $n$ or $n_{0}$. This means that $q$ is close to 1 and the reaction systems stay in an approximate equilibrium state. At a harder scale, the degree of equilibrium decreases due to more disturbance to the equilibrated residual partons in the target particle. Then, one has a larger $q$ and smaller $n$ or $n_{0}$ when compared with those at the softer scale.

Figure 6 shows the excitation functions of related parameters, i.e., the dependencies of (a) $\left\langle p_{T}\right\rangle$, (b) $T_{i}$, (c) $T_{0}$, (d) $n$, (e) $p_{0}$, and (f) $n_{0}$ on the photon-proton center-of-mass 


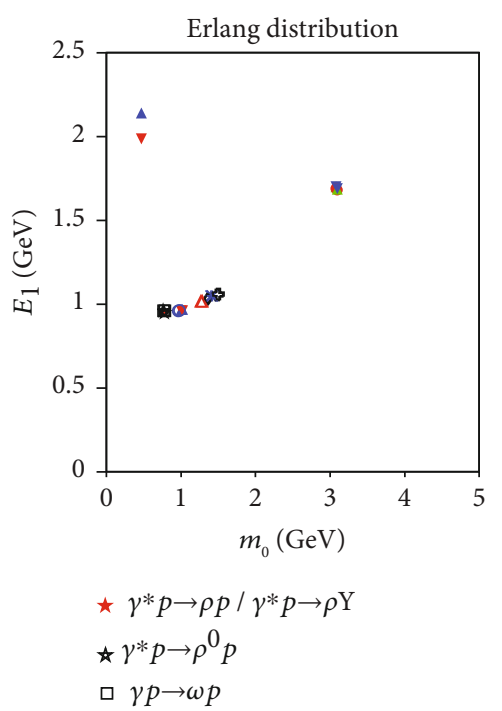

(a)

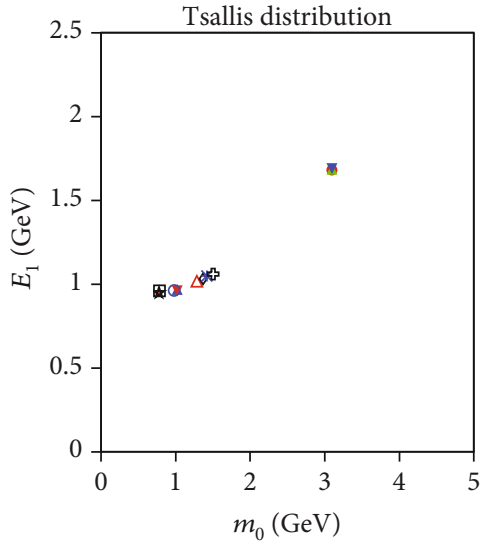

$\Delta \gamma^{*} p \rightarrow \phi p(\mathrm{H} 1) / \gamma^{*} p \rightarrow \phi Y$

$\checkmark \gamma^{*} p \rightarrow \phi p$ (ZEUS)

(b)

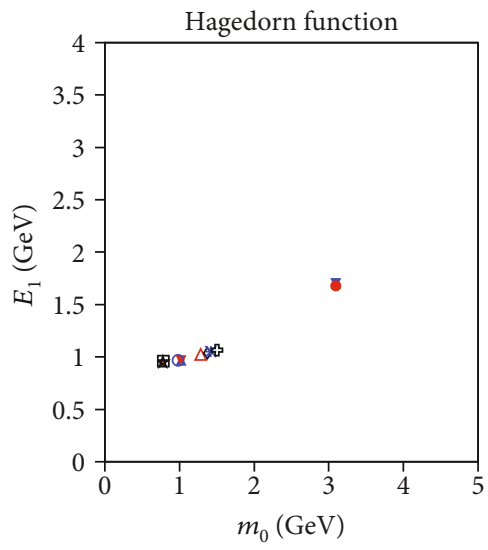

$p p \rightarrow p p$

- $f_{0}(980)$

$\diamond f_{0}(1370)$

\& $f_{0}(1500)$

$\Delta f_{1}(1285)$

* $f_{1}(1420)$

- $\gamma^{*} p \rightarrow J / \psi p$

จ $\gamma p \rightarrow J / \psi \mathrm{p}, \mathrm{Q}^{2}=0.05 \mathrm{GeV}^{2}$

$\Delta \gamma p \rightarrow \mathrm{J} / \psi \mathrm{p}, W=40-160 \mathrm{GeV}$

(c)

FIGURE 4: The dependencies of $E_{1}$ on $m_{0}$ for different two-body reactions which are marked in the panels. (a-c) correspond to the results from the Erlang distribution, Tsallis distribution, and Hagedorn function, respectively.

energy $W$ for $\gamma p \longrightarrow J / \psi p$ reactions. The symbols represent the parameter values extracted from Figure 3 and listed in Tables 1-3. Again, $\left\langle p_{T}\right\rangle=n_{s}\left\langle p_{t}\right\rangle$ from Table 1 and $T_{0}=T$ from Table 2. One can see that $\left\langle p_{T}\right\rangle, T_{i}, T_{0}$, and $p_{0}$ decrease with an increase in $W$, and $n$ and $n_{0}$ increase with an increase in $W$.

In $\gamma p \longrightarrow J / \psi p$ reactions, at a higher center-of-mass energy, the incident photon has a higher energy. Although the emitted $J / \psi$ also has a higher energy, it is more inclined to have a smaller angle. As a comprehensive result, the transverse momentum of $J / \psi$ is smaller, and then, $T_{i}$ and $T_{0}$, which are obtained from the transverse momentum, are also smaller. In addition, larger $n$ and $n_{0}$ at a higher collision energy means more equilibrium due to the shorter collision time and then less disturbance to the equilibrated residual partons in the target particle. This situation is different from nucleus-nucleus collisions in which a cold or spectator nuclear effect has to be considered. 


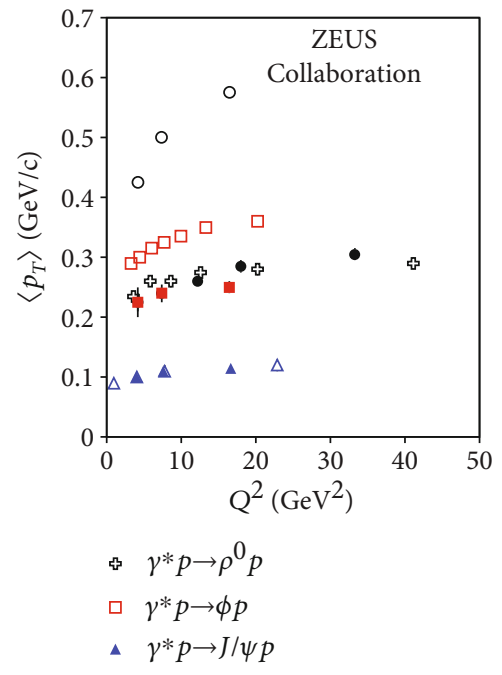

(a)

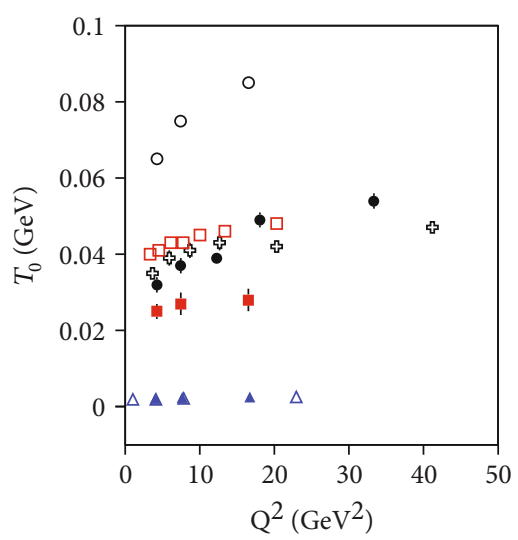

(c)

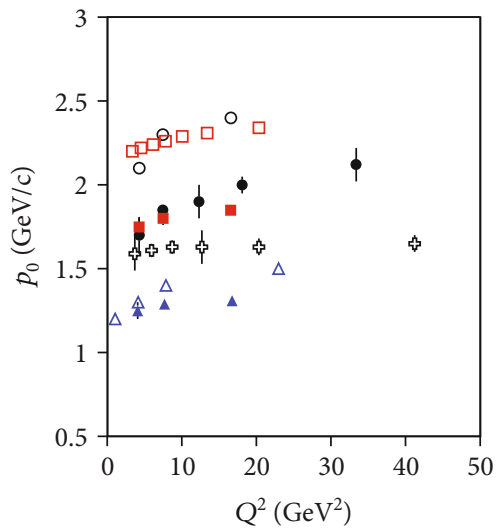

(e)

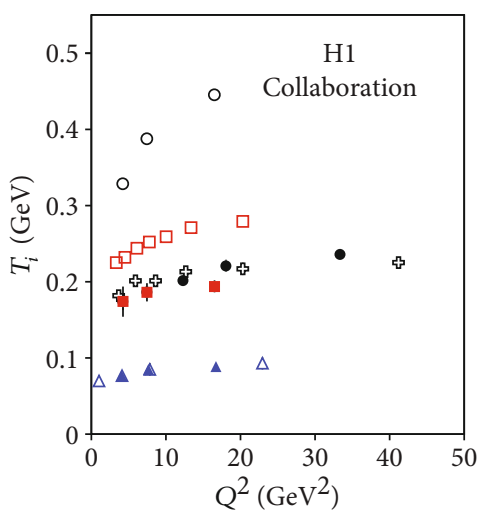

- $\gamma^{*} p \rightarrow \rho p \quad \circ \quad \gamma^{*} p \rightarrow \rho Y$

- $\gamma^{*} p \rightarrow \phi p \quad \Delta \gamma p \rightarrow J / \psi p$

(b)

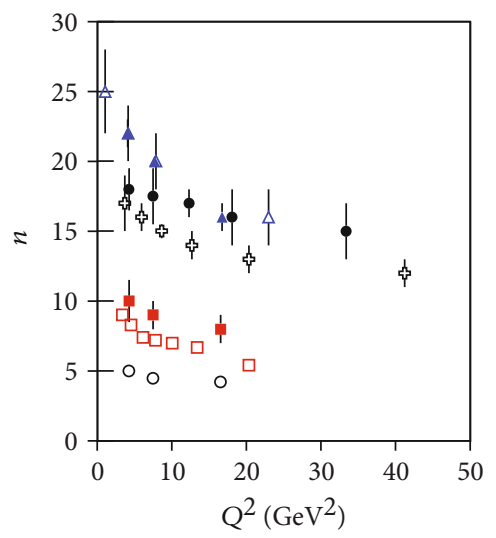

(d)

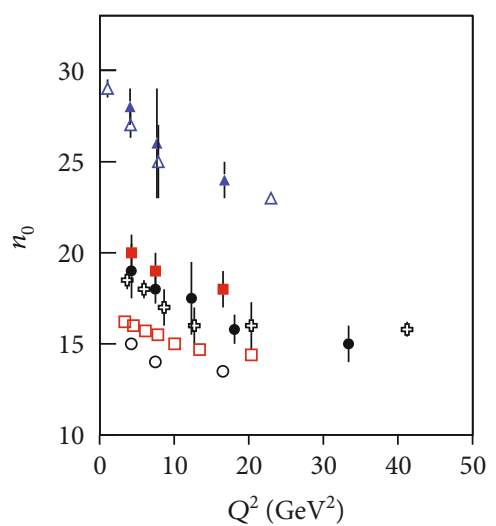

(f)

Figure 5: The dependencies of (a) $\left\langle p_{T}\right\rangle$, (b) $T_{i}$, (c) $T_{0}$, (d) $n$, (e) $p_{0}$, and (f) $n_{0}$ on $Q^{2}$ for different two-body reactions. The symbols represent the parameter values extracted from Figures 1-3 and listed in Tables 1-3. Here, $\left\langle p_{T}\right\rangle=n_{s}\left\langle p_{t}\right\rangle$ from Table 1 and $T_{0}=T$ from Table 2 .

In fact, in nucleus-nucleus collisions, secondary cascade collisions may happen among produced particles and spectator nucleons. The secondary collisions may cause the emission angle to increase and then the transverse momentum to increase. The effect of secondary collisions is more obvious or nearly saturated at a higher energy. In nucleus-nucleus collisions at a lower energy, the system approaches equilib- rium more easily due to a longer interaction time. Conversely, at a higher energy, the system does not approach equilibrium more easily due to the shorter interaction time for secondary collisions.

3.3. Further Discussion. Before the summary and conclusions, we would like to point out that the concept of 

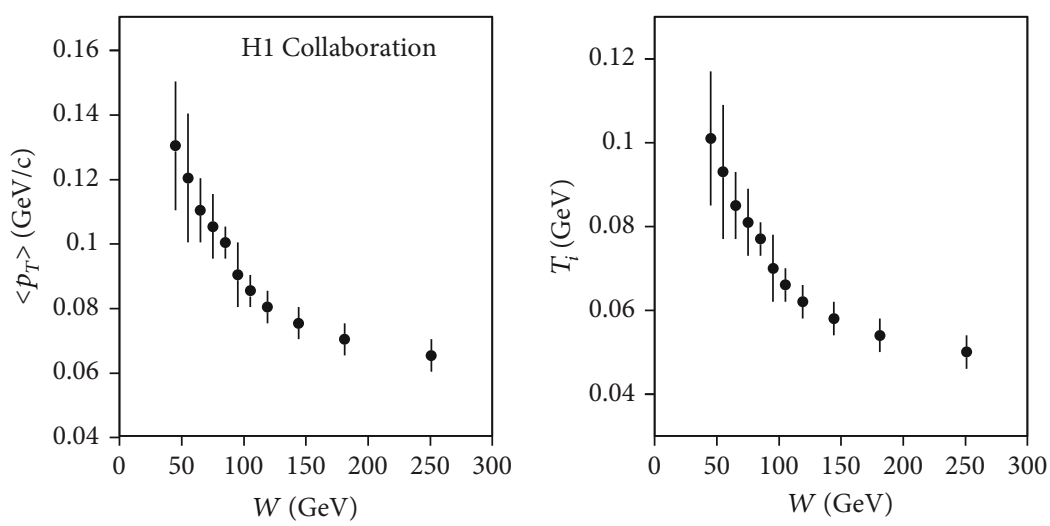

- $\gamma p \rightarrow J / \psi p$

(a)

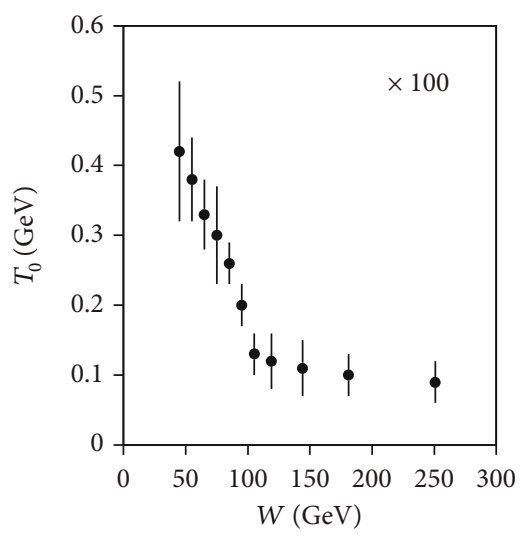

(c)

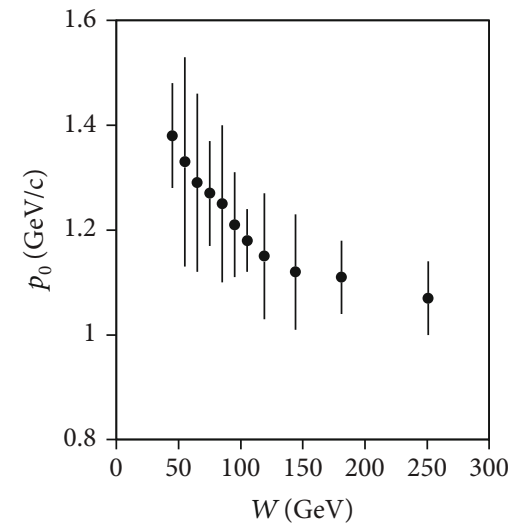

(e) (b)

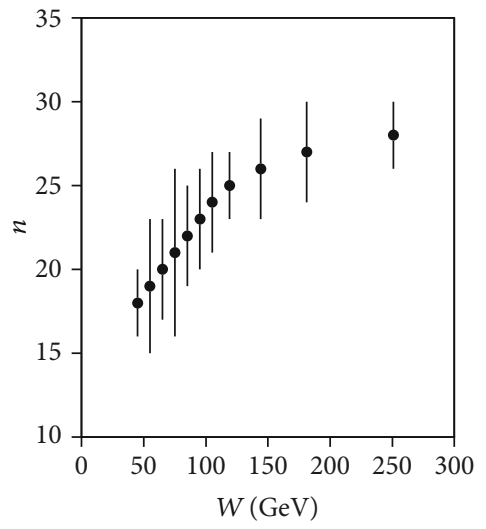

(d)

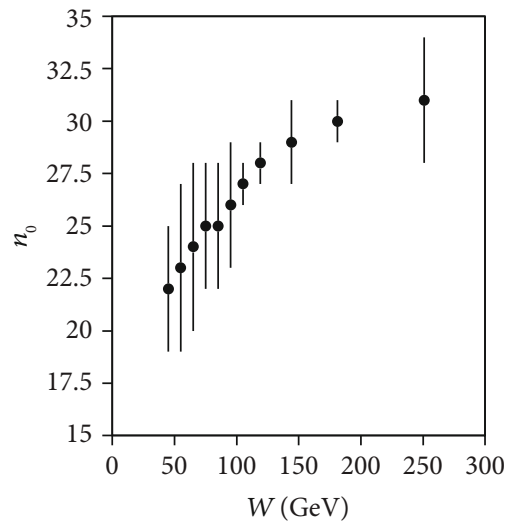

(f)

Figure 6: The dependencies of (a) $\left\langle p_{T}\right\rangle$, (b) $T_{i}$, (c) $T_{0}$, (d) $n$, (e) $p_{0}$, and (f) $n_{0}$ on $W$ for $\gamma p \longrightarrow J / \psi p$ reactions. The symbols represent the parameter values extracted from Figure 3 and listed in Tables $1-3$. Here, $\left\langle p_{T}\right\rangle=n_{s}\left\langle p_{t}\right\rangle$ from Table 1 and $T_{0}=T$ from Table 2 .

temperature used in the present work is valid. Generally, the concept of temperature is used in a large system with multiple particles, which stays in an equilibrium state or approximate (local) equilibrium state. From the macroscopic point of view, the systems of $\gamma^{*} p, \gamma p$, and $p p$ reactions are indeed small. However, we know that there are lots of events under the same condition in the experiments. These events obey the law of grand canonical ensemble in which the concept of temperature is applicable.
Because the same experimental condition is used in statistics, lots of events are in equilibrium if they consist of a large statistical system which can be described by the grand canonical ensemble. Particles in the large statistical system obey the same distribution law such as the same transverse momentum distribution. From the statistical point of view, particle productions in high-energy collisions are a statistical behavior, and the temperature reflects the width of distribution. The higher the temperature is, the wider the distribution is. 
The temperature is also a reflection of the average kinetic energy based on a large statistical system or a single particle. For a single particle, if the distribution law of kinetic energies or transverse momenta is known, the temperature of emission source or interacting system is known, where the source or system means the large thermal source from the ensemble. Generally, we say the temperature of source or system, not saying the temperature of a given particle, from the point of view of statistical significance of temperature. Based on the temperature, we may compare the experimental spectra of different particles in different experiments.

However, different methods have used different distributions or functions, i.e., different "thermometers." To unify these "thermometers" or to find transformations among them, one has to perform quite extensive analysis. Although one may use as far as possible the standard distribution such as the Boltzmann, Fermi-Dirac, or Bose-Einstein distribution to fit the experimental spectra, it is regretful that a single standard distribution cannot fit the experimental spectra very well in general. Naturally, one may use a two-, three-, or even multicomponent standard distribution to fit the experimental spectra, though more parameters are introduced.

In fact, the two-, three-, or multicomponent standard distribution can be fitted satisfactorily by the Tsallis distribution with $q>1$, because the standard distribution is narrower than the Tsallis distribution [68]. In particular, the standard distribution is equivalent to the Tsallis distribution with $q=1$. It is natural to use the Tsallis distribution to replace the standard distribution. That is, one may use the Tsallis distribution with $q>1$ to fit the experimental spectra and obtain the temperature, though the Tsallis temperature is less than the standard one.

As mentioned in the first section and discussed above, some distributions applied in a large collision system can be also applied in a small collision system due to the universality, similarity, or common characteristics existing in highenergy collisions [27-36]. Based on the same reason, some statistical or hydrodynamic models applied in the large system should be also applied in the small system. Of course, lots of events are needed in experiments and high statistics is needed in calculation if performing a Monte Carlo code.

\section{Summary and Conclusions}

In summary, the differential cross-section in the squared momentum transfer of $\rho, \rho^{0} \omega, \phi, f_{0}(980), f_{1}(1285), f_{0}($ $1370), f_{1}(1420), f_{0}(1500)$, and $J / \psi$ produced in $\gamma^{*} p, \gamma p$, and $p p$ collisions has been analyzed by the Monte Carlo calculations in which the Erlang distribution, Tsallis distribution, and Hagedorn function (inverse power law) are separately used to describe the transverse momentum spectra of the emitted particles. In most cases, the model results are approximately in agreement with the experimental data measured by the H1, ZEUS, and WA102 Collaborations. In some cases, the fits show qualitatively the data tendencies. The values of the initial- and final-state temperatures and other related parameters are extracted from the fitting process. The squared photon virtuality
$Q^{2}$ and center-of-mass energy $W$-dependent parameters are obtained.

With an increase in $Q^{2}$, the quantities $\left\langle p_{T}\right\rangle, T_{i}, T_{0}$, and $p_{0}$ increase generally, and the quantities $n$ and $n_{0}$ decrease significantly. $Q^{2}$ is a reflection of a hard scale of reaction. A harder scale results in a higher excitation degree and then a larger $\left\langle p_{T}\right\rangle, T_{i}$, and $T_{0}$. In most cases, the reaction system can be regarded as an equilibrium state. At a harder scale (larger $Q^{2}$ ), the degree of equilibrium decreases due to more disturbance to the equilibrated residual partons in the target particle, though the degree of excitation is high.

With the increase of $W$, the quantities $\left\langle p_{T}\right\rangle, T_{i}, T_{0}$, and $p_{0}$ decrease, and the quantities $n$ and $n_{0}$ increase. In $\gamma p \longrightarrow J / \psi p$ reactions at a high energy, the emitted $J / \psi$ is more inclined to have a small angle and hence small $p_{T}, T_{i}$, and $T_{0}$. In addition, the system stays in a state with a higher degree of equilibrium at high energy due to less disturbance to the equilibrated residual partons in the target particle. This situation is different from nucleus-nucleus collisions in which the influence of a cold or spectator nuclear effect is existent.

\section{Data Availability}

This manuscript has no associated data or the data will not be deposited. (Authors' comment: the data used to support the findings of this study are included within the article and are cited at relevant places within the text as references.)

\section{Ethical Approval}

The authors declare that they are in compliance with ethical standards regarding the content of this paper.

\section{Disclosure}

The funding agencies have no role in the design of the study; in the collection, analysis, or interpretation of the data; in the writing of the manuscript; or in the decision to publish the results.

\section{Conflicts of Interest}

The authors declare that there are no conflicts of interest regarding the publication of this paper.

\section{Acknowledgments}

The work of Q.W. and F.H.L. was supported by the National Natural Science Foundation of China under Grant Nos. 12047571, 11575103, and 11947418; the Scientific and Technological Innovation Programs of Higher Education Institutions in Shanxi (STIP) under Grant No. 201802017; the Shanxi Provincial Natural Science Foundation under Grant No. 201901D111043; and the Fund for Shanxi "1331 Project" Key Subjects Construction. The work of K.K.O. was supported by the Ministry of Innovative Development of the Republic of Uzbekistan within the fundamental project on 
analysis of open data on heavy-ion collisions at RHIC and LHC.

\section{References}

[1] H. Wang, J.-H. Chen, Y.-G. Ma, and S. Zhang, "Charm hadron azimuthal angular correlations in $\mathrm{Au}+\mathrm{Au}$ collisions at $\sqrt{s_{\mathbb{N}}}$ $=200 \mathrm{GeV}$ from parton scatterings," Nuclear Science and Techniques, vol. 30, no. 12, p. 185, 2019.

[2] T.-Z. Yan, S. Li, Y.-N. Wang, F. Xie, and T.-F. Yan, "Yield ratios and directed flows of light particles from proton-rich nuclei-induced collisions," Nuclear Science and Techniques, vol. 31, p. 15, 2019.

[3] M. Fisli and N. Mebarki, "Top quark pair-production in noncommutative standard model," Advances in High Energy Physics, vol. 2020, Article ID 7279627, 6 pages, 2020.

[4] X.-W. He, F.-M. Wu, H.-R. Wei, and B.-H. Hong, "Energydependent chemical potentials of light hadrons and quarks based on transverse momentum spectra and yield ratios of negative to positive particles," Advances in High Energy Physics, vol. 2020, Article ID 1265090, 19 pages, 2020.

[5] M. Waqas and B.-C. Li, "Kinetic freeze-out temperature and transverse flow velocity in Au-Au collisions at RHIC-BES energies," Advances in High Energy Physics, vol. 2020, Article ID 1787183, 14 pages, 2020.

[6] Z.-B. Tang, W.-M. Zha, and Y.-F. Zhang, "An experimental review of open heavy flavor and quarkonium production at RHIC," Nuclear Science and Techniques, vol. 31, no. 8, p. 81, 2020.

[7] C. Shen and L. Yan, "Recent development of hydrodynamic modeling in heavy-ion collisions," Nuclear Science and Techniques, vol. 31, no. 12, p. 122, 2020.

[8] H. Yu, D.-Q. Fang, and Y.-G. Ma, "Investigation of the symmetry energy of nuclear matter using isospin-dependent quantum molecular dynamics," Nuclear Science and Techniques, vol. 31, no. 6, p. 61, 2020.

[9] S. Bhaduri, A. Bhaduri, and D. Ghosh, "Study of di-muon production process in pp collision in CMS data from symmetry scaling perspective," Advances in High Energy Physics, vol. 2020, Article ID 4510897, 17 pages, 2020.

[10] A. N. Tawfik, "Out-of-equilibrium transverse momentum spectra of pions at LHC energies," Advances in High Energy Physics, vol. 2020, Article ID 4604608, 7 pages, 2019.

[11] J. K. Nayak, J. Alam, S. Sarkar, and B. Sinha, "Measuring initial temperature through a photon to dilepton ratio in heavy-ion collisions," Journal of Physics G, vol. 35, no. 10, article 104161, 2008.

[12] A. Adare, S. Afanasiev, C. Aidala et al., "Enhanced production of direct photons in $\mathrm{Au}+\mathrm{Au}$ collisions at $\sqrt{ } s_{N N}=200 \mathrm{GeV}$ and implications for the initial temperature," Physical Review Letters, vol. 104, article 132301, 2010.

[13] M. Csanád and I. Májer, "Initial temperature and EoS of quark matter via direct photons," Physics of Particles and Nuclei Letters, vol. 8, no. 9, pp. 1013-1015, 2011.

[14] M. Csanád and I. Májer, "Equation of state and initial temperature of quark gluon plasma at RHIC," Central European Journal of Physics, vol. 10, pp. 850-857, 2012.

[15] R. A. Soltz, I. Garishvili, M. Cheng et al., "Constraining the initial temperature and shear viscosity in a hybrid hydrodynamic model of $\sqrt{s_{\mathbb{N}}}=200 \mathrm{GeV}$ Au+Au collisions using pion spectra, elliptic flow, and femtoscopic radii," Physical Review C, vol. 87, no. 4 , article $044901,2013$.

[16] M. Waqas and F.-H. Liu, "Initial, effective, and kinetic freezeout temperatures from transverse momentum spectra in highenergy proton(deuteron)-nucleus and nucleus-nucleus collisions," The European Physical Journal Plus, vol. 135, no. 2, p. 147, 2020.

[17] J. Cleymans and M. W. Paradza, "Tsallis statistics in high energy physics: chemical and thermal freeze-outs," Physics, vol. 2, no. 4, pp. 654-664, 2020.

[18] L.-L. Li and F.-H. Liu, "Kinetic freeze-out properties from transverse momentum spectra of pions in high energy proton-proton collisions," Physics, vol. 2, no. 2, pp. 277-308, 2020.

[19] F.-H. Liu and J.-S. Li, "Isotopic production cross section of fragments in ${ }^{56} \mathrm{Fe}+p$ and ${ }^{136} \mathrm{Xe}\left({ }^{124} \mathrm{Xe}\right)+\mathrm{Pb}$ reactions over an energy range from $300 \mathrm{~A}$ to $1500 \mathrm{~A} \mathrm{MeV}$," Physical Review C, vol. 78, no. 4, article 044602, 2008.

[20] F.-H. Liu, "Unified description of multiplicity distributions of final-state particles produced in collisions at high energies," Nuclear Physics A, vol. 810, no. 1-4, pp. 159-172, 2008.

[21] F.-H. Liu, Y.-Q. Gao, T. Tian, and B.-C. Li, "Unified description of transverse momentum spectrums contributed by soft and hard processes in high-energy nuclear collisions," The European Physical Journal A, vol. 50, no. 6, p. 94, 2014.

[22] C. Tsallis, "Possible generalization of Boltzmann-Gibbs statistics," Journal of Statistical Physics, vol. 52, no. 1-2, pp. 479487, 1988.

[23] B. I. Abelev, J. Adams, M. M. Aggarwal et al., "Strange particle production in $p+p$ collisions at $\sqrt{s}=200 \mathrm{GeV}$," Physical Review C, vol. 75, article 064901, 2007.

[24] R. Hagedorn, "Multiplicities, $p_{T}$ distributions and the expected hadron $\longrightarrow$ quark-gluon phase transition," La Rivista del Nuovo Cimento, vol. 6, no. 10, pp. 1-50, 1983.

[25] B. Abelev, J. Adam, D. Adamová et al., "Production of $\Sigma(1385)^{ \pm}$and $\Xi(1530)^{0}$ in proton-proton collisions at $\sqrt{s}=7$ TeV," The European Physical Journal C, vol. 75, no. 1, pp. 119, 2015.

[26] N.-S. Zhang, Particle Physics (Volume I), Science Press, Beijing, China, 1986.

[27] E. K. G. Sarkisyan and A. S. Sakharov, "Multihadron production features in different reactions," AIP Conference Proceedings, vol. 828, pp. 35-41, 2006.

[28] E. K. G. Sarkisyan and A. S. Sakharov, "Relating multihadron production in hadronic and nuclear collisions," The European Physical Journal C, vol. 70, no. 3, pp. 533-541, 2010.

[29] A. N. Mishra, R. Sahoo, E. K. G. Sarkisyan, and A. S. Sakharov, "Effective-energy budget in multiparticle production in nuclear collisions," The European Physical Journal C, vol. 74, no. 11, article $3147,2014$.

[30] E. K. G. Sarkisyan, A. N. Mishra, R. Sahoo, and A. S. Sakharov, "Multihadron production dynamics exploring the energy balance in hadronic and nuclear collisions," Physical Review D, vol. 93, no. 5, article 054046, 2016.

[31] E. K. G. Sarkisyan, A. N. Mishra, R. Sahoo, and A. S. Sakharov, "Centrality dependence of midrapidity density from $\mathrm{GeV}$ to $\mathrm{TeV}$ heavy-ion collisions in the effective-energy universality picture of hadroproduction," Physical Review D, vol. 94, no. 1, article 011501, 2016.

[32] E. K. G. Sarkisyan, A. N. Mishra, R. Sahoo, and A. S. Sakharov, "Effective-energy universality approach describing total 
multiplicity centrality dependence in heavy-ion collisions," $E P L$, vol. 127, no. 6, article 62001, 2019.

[33] A. N. Mishra, A. Ortiz, and G. Paic, "Intriguing similarities of high- $p_{T}$ particle production between $p p$ and $A-A$ collisions," Physical Review C, vol. 99, no. 3, article 034911, 2019.

[34] P. Castorina, S. Plumari, and H. Satz, "Universal strangeness production in hadronic and nuclear collisions," International Journal of Modern Physics E, vol. 25, no. 8, article 1650058, 2016.

[35] P. Castorina, A. Iorio, D. Lanteri, H. Satz, and M. Spousta, "Universality in high energy collisions of small and large systems," in Proceedings of the 40th International Conference on High Energy physics - ICHEP2020, vol. 390no. ICHEP2020, p. 537, Prague, Czech Republic, 2020, https://arxiv.org/abs/ 2012.12514.

[36] P. Castorina, A. Iorio, D. Lanteri, H. Satz, and M. Spousta, "Universality in hadronic and nuclear collisions at high energy," Physical Review C, vol. 101, no. 5, article 054902, 2020.

[37] The H1 Collaboration, F. D. Aaron, M. A. Martin et al., "Diffractive electroproduction of $\rho$ and $\phi$ mesons at HERA," Journal of High Energy Physics, vol. 2010, no. 5, p. 32, 2010.

[38] H1 Collaboration, "Elastic $J / \psi$ production at HERA," The European Physical Journal C, vol. 46, pp. 585-603, 2006.

[39] ZEUS Collaboration, "Exclusive $\rho^{0}$ production in deep inelastic scattering at HERA," PMC Physics A, vol. 1, p. 6, 2007.

[40] ZEUS Collaboration, "Measurement of elastic $\omega$ photoproduction at HERA ZEUS Collaboration," Zeitschrift für Physik C, vol. 73, pp. 73-84, 1997.

[41] ZEUS Collaboration, "Exclusive electroproduction of $\phi$ mesons at HERA," Nuclear Physics B, vol. 718, pp. 3-31, 2005.

[42] ZEUS Collaboration, "Exclusive electroproduction of $\mathrm{J} / \psi$ mesons at HERA," Nuclear Physics B, vol. 695, pp. 3-37, 2004.

[43] WA102 Collaboration, "A coupled channel analysis of the centrally produced $\mathrm{K}^{+} \mathrm{K}^{-}$and $\pi^{+} \pi^{-}$final states in pp interactions at 450 GeV/c," Physics Letters B, vol. 462, pp. 462-470, 1999.

[44] D. Barberis, W. Beusch, F. G. Binon et al., "A measurement of the branching fractions of the $\mathrm{f}_{1}(1285)$ and $\mathrm{f}_{1}(1420)$ produced in central pp interactions at $450 \mathrm{GeV} / \mathrm{c}$," Physics Letters $B$, vol. 440, no. 1-2, pp. 225-232, 1998.

[45] L. J. Gutay, A. S. Hirsch, R. P. Scharenberg, B. K. Srivastava, and C. Pajares, "De-confinement in small systems: clustering of color sources in high multiplicity $\bar{p} p$ collisions at $\sqrt{ } s=1.8$ TeV," International Journal of Modern Physics E, vol. 24, no. 12, article 1550101, 2015.

[46] R. P. Scharenberg, B. K. Srivastava, C. Pajares, and B. K. Srivastava, "Exploring the initial stage of high multiplicity protonproton collisions by determining the initial temperature of the quark-gluon plasma," Physical Review D, vol. 100, no. 11, article 114040, 2019.

[47] P. Sahoo, S. De, S. K. Tiwari, and R. Sahoo, "Energy and centrality dependent study of deconfinement phase transition in a color string percolation approach at RHIC energies," The European Physical Journal A, vol. 54, no. 8, p. 136, 2018.

[48] Q. Wang and F.-H. Liu, "Excitation function of initial temperature of heavy flavor quarkonium emission source in high energy collisions," Advances in High Energy Physics, vol. 2020, Article ID 5031494, 31 pages, 2020.

[49] E. Schnedermann, J. Sollfrank, and U. Heinz, “Thermal phenomenology of hadrons from 200A GeV S+S collisions," Physical Review C, vol. 48, no. 5, pp. 2462-2475, 1993.
[50] STAR Collaboration, "Systematic measurements of identified particle spectra in $\mathrm{pp}, \mathrm{d}+\mathrm{Au}$, and $\mathrm{Au}+\mathrm{Au}$ collisions at the STAR detector," Physical Review C, vol. 79, article 034909, 2009.

[51] STAR Collaboration, "Identified particle production, azimuthal anisotropy, and interferometry measurements in $\mathrm{Au}+\mathrm{Au}$ collisions at $\sqrt{ } s_{N N}=9.2 \mathrm{GeV}$," Physical Review C, vol. 81, article 024911, 2010.

[52] Z. B. Tang, Y. C. Xu, L. J. Ruan, G. van Buren, F. Q. Wang, and Z. B. Xu, "Spectra and radial flow in relativistic heavy ion collisions with Tsallis statistics in a blast-wave description," Physical Review C, vol. 79, no. 5, article 051901, 2009.

[53] P. K. Khandai, P. Sett, P. Shukla, and V. Singh, "System size dependence of hadron $p_{T}$ spectra in $\mathrm{p}+\mathrm{p}$ and $\mathrm{Au}+\mathrm{Au}$ collisions at $\sqrt{ } s_{N N}=200 \mathrm{GeV}$," Journal of Physics G, vol. 41, no. 2, article 025105, 2014.

[54] K. K. Olimov, S. Z. Kanokova, K. Olimov et al., “Average transverse expansion velocities and global freeze-out temperatures in central $\mathrm{Cu}+\mathrm{Cu}, \mathrm{Au}+\mathrm{Au}$, and $\mathrm{Pb}+\mathrm{Pb}$ collisions at high energies at RHIC and LHC," Modern Physics Letters A, vol. 35, no. 14, article 2050115, 2020.

[55] K. K. Olimov, S. Z. Kanokova, A. K. Olimov et al., "Combined analysis of midrapidity transverse momentum spectra of the charged pions and kaons, protons and antiprotons in $\mathrm{p}+\mathrm{p}$ and $\mathrm{Pb}+\mathrm{Pb}$ collisions at $\left(s_{n n}\right)^{1 / 2}=2.76$ and $5.02 \mathrm{TeV}$ at the LHC," Modern Physics Letters A, vol. 35, no. 29, article 2050237, 2020.

[56] K. K. Olimov, A. Iqbal, and S. Masood, "Systematic analysis of midrapidity transverse momentum spectra of identified charged particles in $\mathrm{p}+\mathrm{p}$ collisions at $\left(s_{n n}\right)^{1 / 2}=2.76,5.02$, and $7 \mathrm{TeV}$ at the LHC," International Journal of Modern Physics A, vol. 35, no. 27, article 2050167, 2020.

[57] K. K. Olimov, K. I. Umarov, A. Iqbal, S. Masood, and F.-H. Liu, "Analysis of midrapidity transverse momentum distributions of the charged pions and kaons, protons and antiprotons in $\mathrm{p}+\mathrm{p}$ collisions at $\left(s_{n n}\right)^{1 / 2}=2.76,5.02$, and $7 \mathrm{TeV}$ at the LHC," in Proceedings of International Conference "Fundamental and Applied Problems of Physics", pp. 78-83, Tashkent, Uzbekistan, September 2020.

[58] S. Takeuchi, K. Murase, T. Hirano, P. Huovinen, and Y. Nara, "Effects of hadronic rescattering on multistrange hadrons in high-energy nuclear collisions," Physical Review C, vol. 92, no. 4, article 044907, 2015.

[59] H. Heiselberg and A.-M. Levy, "Elliptic flow and HanburyBrown-Twiss correlations in noncentral nuclear collisions," Physical Review C, vol. 59, no. 5, pp. 2716-2727, 1999.

[60] U. W. Heinz, "Concepts of heavy-ion physics," in Lecture Notes for Lectures Presented at the 2nd CERN-LatinAmerican School of High-Energy Physics, San Miguel Regla, Mexico, June 2003https://arxiv.org/abs/hep-ph/0407360.

[61] R. Russo, Measurement of $\mathrm{D}^{+}$meson production in $\mathrm{p}-\mathrm{Pb}$ collisions with the ALICE detector, [Ph.D. thesis], Universita degli Studi di Torino, Italy, 2015.

[62] H.-L. Lao, F.-H. Liu, B.-C. Li, and M.-Y. Duan, "Kinetic freezeout temperatures in central and peripheral collisions: which one is larger?," Nuclear Science and Techniques, vol. 29, no. 6, p. 82, 2018.

[63] H.-L. Lao, F.-H. Liu, B.-C. Li, M.-Y. Duan, and R. A. Lacey, "Examining the model dependence of the determination of kinetic freeze-out temperature and transverse flow velocity in small collision system," Nuclear Science and Techniques, vol. 29, no. 11, p. 164, 2018. 
[64] H.-R. Wei, F.-H. Liu, and R. A. Lacey, "Kinetic freeze-out temperature and flow velocity extracted from transverse momentum spectra of final-state light flavor particles produced in collisions at RHIC and LHC," The European Physical Journal $A$, vol. 52, no. 4, p. 102, 2016.

[65] H.-L. Lao, H.-R. Wei, and F.-H. Liu, "An evidence of massdependent differential kinetic freeze-out scenario observed in $\mathrm{Pb}-\mathrm{Pb}$ collisions at $2.76 \mathrm{TeV}$," The European Physical Journal $A$, vol. 52, no. 7, p. 203, 2016.

[66] H.-R. Wei, F.-H. Liu, and R. A. Lacey, "Disentangling random thermal motion of particles and collective expansion of source from transverse momentum spectra in high energy collisions," Journal of Physica G, vol. 43, no. 12, article 125102, 2016.

[67] A. D. Martin and M. G. Ryskin, "The photon PDF of the proton," The European Physical Journal C, vol. 74, no. 9, article 3040, 2014.

[68] F.-H. Liu, Y.-Q. Gao, and H.-R. Wei, "On descriptions of particle transverse momentum spectra in high energy collisions," Advances in High Energy Physics, vol. 2014, Article ID 293873, 12 pages, 2014. 